\title{
4. UNDERWAY GEOPHYSICS COLLECTED ON DEEP SEA DRILLING PROJECT LEG 68 ${ }^{1}$
}

\author{
James V. Gardner, U.S. Geological Survey, Menlo Park, California
}

\section{INTRODUCTION}

Glomar Challenger departed Willemstad, Curacao, on Leg 68 on 13 August 1979, and arrived at the Caribbean side of the Panama Canal on 28 August. It departed from the Pacific side of the Canal on 3 September, completing the leg at Salinas, Ecuador, on $18 \mathrm{Sep}$ tember. The trackline of the cruise is shown in Figure 1.

Seismic-reflection profiles $(3.5 \mathrm{kHz}$ and air gun) and magnetics data were continuously recorded along the transit. The $3.5 \mathrm{kHz}$ data are not presented here because of the volume of data involved. They can be viewed upon request at the Deep Sea Drilling Project (DSDP) West Coast Repository, Scripps Institution of Oceanography, La Jolla, California. The air-gun system utilized a 5 -in. ${ }^{3}$ and a 40 -in. ${ }^{3}$ air gun, fired simultaneously, and data were recorded after passing through an 80 to 160 $\mathrm{Hz}$ band-pass filter. The master recorder triggered the system at 10 -s intervals (not presented here), and a slave was time-delayed and recorded at 5-s intervals (presented here). (Time increases from left to right on all figures.) Table 1 lists navigation data reduced from the primary satellite fixes and the ship's gyro and speed $\log$. The navigation data include day; month; year; time in Greenwich time; latitude and longitude in degrees, minutes, and tenths of minutes (a negative longitude is west); cumulative distance along the track in nautical miles; actual speed (in knots) and course calculated from satellite fixes; drift speed (in knots) and heading calculated from satellite fixes and dead reckoning; dead reckoning speed (in knots) and course from the ship's speed log and gyro; and a comment column with miscellaneous events.

Figures 2 through 5 show bathymetry and magnetic anomaly plotted in profile as functions of time, distance, latitude, and longitude with distance as the linear function. All depths are plotted in uncorrected meters. The seismic-reflection profiles are shown in Figures 7 through 29.

\section{MAGNETICS}

Our magnetics data from the Caribbean transect show only small-amplitude anomalies with a notable lack of fluctuations over the continental margin (Fig. 2).

The magnetics data from our Pacific traverse are shown in Figures 3 through 5. The scientists of Leg 9 (Hays et al., 1972) describe Site 83 as being on the east flank of the East Pacific Rise (EPR). Our magnetics

\footnotetext{
${ }^{1}$ Prell, W. L., Gardner, J. V., et al., Init. Repts. DSDP, 68: Washington (U.S. Govt. Printing Office).
}

data, however, support the alternate interpretation (implied by van Andel et al., 1975, fig. 7) that Sites 83 and 503 (within seven nautical miles of each other) are both located on the north flank of the Galapagos Ridge. If our track had traversed the eastern flank of the EPR, large-amplitude magnetic anomalies should have been recorded as a result of our crossing roughly perpendicular to the magnetic lineations. If, on the other hand, our track were along the north flank of the Galapagos Ridge, then we would have been parallel to the magnetic lineations, and the magnetic anomalies should have been subdued and not well defined. In fact, the magnetic anomalies along our track to Site 503 were subdued and ill defined (Fig. 4). The trackline shows high-amplitude magnetic anomalies from the time we departed Site 503-similar to the pattern predicted if we were over the Galapagos Ridge. Figure 4 shows the magnetic anomalies prior to and after coring operationis at Site 503. The distinct difference in character of the magnetic anomalies coincides with the change from approach to departure courses. This evidence strongly suggests that we located Site 503, and hence also Site 83 (Leg 9), on the north flank of Galapagos Ridge.

\section{SEISMIC REFLECTION PROFILES}

The bathymetry of the Caribbean region traversed on Leg 68 is shown in Figure 6A. The seismic-reflection system was turned on after about $20 \mathrm{hr}$. of a shakedown cruise out of Curacao. Figures 7 through 9 show a traverse from the upper continental slope to the Colombia Basin. A large basin is clearly shown to be formed behind a large, uplifted(?) block. The channel at 2330 hr. on 14 August is part of an unnamed canyon system shown on the bathymetric chart (Fig. 6A) to incise the shelf break and traverse northwesterly to eventually feed onto the Colombia Basin. The large uplifted(?) block appears to be part of what is probably a major tectonic shear underlying the continental margin. Figures 9 through 11 show a crossing onto the Colombia Basin (Fig. 9A), then over the Magdalena Fan (Figs. 9B, 10, 11). The flat, smooth, channelized surface of the Colombia Basin is contrasted by the hummocky, non-channelized surface of the Magdalena Fan. The diffuse reflector at about 0.2- to 0.3-s reflection time in Figures 10 through 13 is the middle Pliocene and Miocene volcanogenic turbidites recovered at Site 154 (Edgar et al., 1973). Figures 11 and 12 show our crossing onto what Holcombe (1975) calls Mono Ridge, the feature drilled at Sites 154 and 502. The location of Site 502 is marked on Figure 12. Our traverse from Site 502 to Panama (Fig. 13) shows the post-Miocene turbidites of the Pana- 


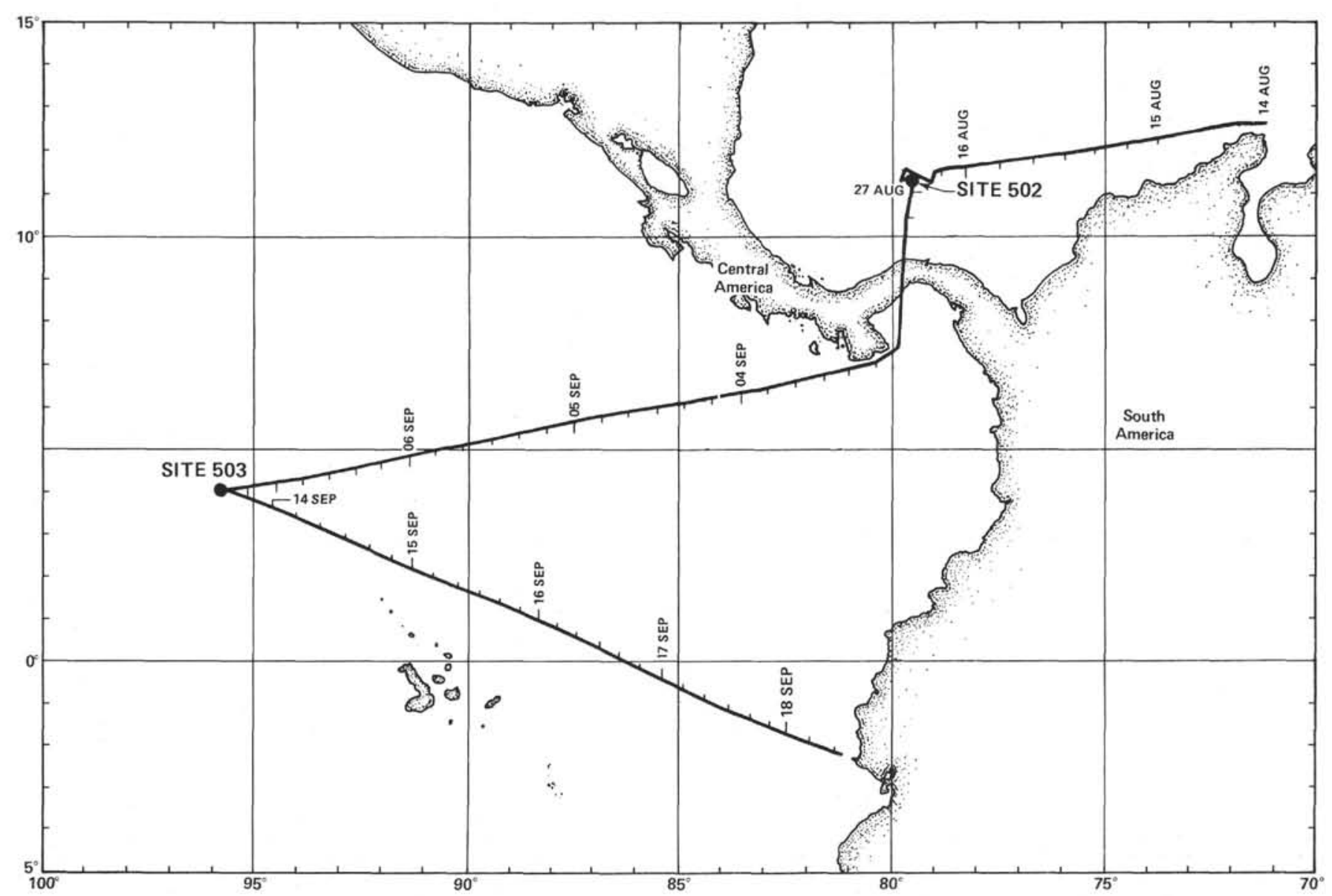

Figure 1. Tracklines for Leg 68 of Glomar Challenger. Tick marks along track are hourly marks. (Day/month point to $0000 \mathrm{Z}$ hr.)

ma Plain onlapping the uplifted block where Site 502 was drilled.

The bathymetry of the portion of the eastern equatorial Pacific traversed during Leg 68 is shown on Figure 6B. The seismic-reflection system was not deployed until the ship was west of the Azuero Peninsula of Panama because of heavy ship traffic. We traversed a section of very rough topography with evidence of numerous deep canyons and faults (Fig. 14) and then crossed Coiba Ridge (Fig. 15). Coiba Ridge has a very thick, acoustically transparent section on the eastern flank and a steep escarpment on its western flank. We then crossed over a region of steep, faulted terrain with a large seamount and approached Cocos Ridge. The area between Coiba Ridge and the large seamount appears to be a large graben (Fig. 15). The large seamount has dammed sediment that slumped from Cocos Ridge.

Cocos Ridge (Figs. 15-18) appears as a large bathymetric high with a thick sediment blanket having many slumps along the edges and faults throughout. $\mathrm{Nu}$ merous projections of acoustic basement occur over the Ridge and large, steep seamounts are more numerous along the western margin (Fig. 18) than elsewhere on the Ridge.

We continued our traverse and crossed the southern section of the Guatemala Basin along the boundary with the north flank of the Galapagos Ridge (Figs. 18-24).
This area has a relatively thick section of sediment with numerous faults, resulting in a well-defined fault-controlled topography. Several large-scale channels are seen on Figures 18 through 20 leading from the Galapagos Ridge to the Guatemala Basin. Site 503 is located on a relatively smooth, thick section of sediment, which is acoustically stratified but which grades into acoustically transparent sediment toward the east. The transparent section is probably composed of slumped and otherwise deformed sediment.

Our trip from Site 503 to Salinas, Ecuador, went over the Galapagos Ridge, which shows relatively thick, faulted sediment between basement highs (Figs. 22, 23), but the sediment section becomes thinner near the Galapagos Islands (Figs. 24, 25). The sediment section becomes thicker and less faulted southeast of the Galapagos Islands (Figs. 25, 26).

The track continues to the northwest escarpment on the southern end of Carnegie Ridge. Sediment on this ridge has variable thickness and is in places highly faulted (Fig. 27); in other places it is very smooth (Fig. 28). Our track took us from Carnegie Ridge across the northern portion of the Peru-Chile Trench (Fig. 29). Unfortunately, we did not get a good profile of the trench floor. Sediment is clearly slumped and faulted on the outer trench wall and a small perched basin is seen on the inner trench wall. The seismic systems were turned 
Table 1. Navigation data, Leg 68.

\begin{tabular}{|c|c|c|c|c|c|c|c|c|c|c|c|c|c|c|c|}
\hline \multirow[b]{2}{*}{ Day } & \multirow[b]{2}{*}{ Month } & \multirow[b]{2}{*}{ Year } & & Lat & ude & Long & tude & & & tual & & rift & Dead R & eckoning & \\
\hline & & & Time & Degree & Minute & Degree & Minute & Distance & Speed & Course & Speed & Heading & Speed & Course & Comments $^{\mathrm{a}}$ \\
\hline 14 & 8 & 1979 & $\cdot 1050$ & 12 & 43.0 & -71 & 26.0 & 1636.2 & 0.4 & 163 & 0.4 & 163 & 0.0 & 0 & DEP \\
\hline 14 & 8 & 1979 & 1050 & 12 & 43.0 & -71 & 26.0 & 1636.2 & 6.7 & 265 & 0.4 & 163 & 6.8 & 268 & $\mathrm{U} / \mathrm{W}$ \\
\hline 14 & 8 & 1979 & 1055 & 12 & 43.0 & -71 & 26.6 & 1636.7 & 8.9 & 266 & 0.4 & 163 & 9.0 & 268 & $\mathrm{C} / \mathrm{S}$ \\
\hline 14 & 8 & 1979 & 1109 & 12 & 42.8 & -71 & 28.7 & 1638.8 & 9.0 & 259 & 0.4 & 163 & 9.0 & 261 & $\mathrm{C} / \mathrm{C}$ \\
\hline 14 & 8 & 1979 & 1116 & 12 & 42.6 & -71 & 29.7 & 1639.9 & 9.8 & 259 & 0.4 & 163 & 9.8 & 261 & $\mathrm{C} / \mathrm{S}$ \\
\hline 14 & 8 & 1979 & 1142 & 12 & 41.8 & -71 & 34.0 & 1644.1 & 9.0 & 259 & 0.4 & 163 & 9.0 & 261 & $\mathrm{C} / \mathrm{S}$ \\
\hline 14 & 8 & 1979 & $\bullet 1202$ & 12 & 41.2 & -71 & 37.0 & 1647.1 & 9.2 & 261 & 0.2 & 254 & 9.0 & 261 & SATL \\
\hline 14 & 8 & 1979 & $\bullet 1320$ & 12 & 39.3 & -71 & 49.1 & 1659.0 & 9.9 & 261 & 0.9 & 256 & 9.0 & 261 & SATL \\
\hline 14 & 8 & 1979 & 1515 & 12 & 36.2 & -72 & 8.3 & 1678.1 & 10.7 & 259 & 0.9 & 256 & 9.8 & 259 & $\mathrm{C} / \mathrm{C}$ \\
\hline 14 & 8 & 1979 & *1526 & 12 & 35.8 & -72 & 10.3 & 1680.0 & 10.2 & 257 & 0.5 & 214 & 9.8 & 259 & SATL \\
\hline 14 & 8 & 1979 & • 1710 & 12 & 31.8 & -72 & 27.9 & 1697.7 & 10.8 & 259 & 1.0 & 259 & 9.8 & 259 & SATL \\
\hline 14 & 8 & 1979 & $* 2136$ & 12 & 22.7 & -73 & 15.9 & 1745.4 & 10.6 & 260 & 0.8 & 275 & 9.8 & 259 & SATL \\
\hline 14 & 8 & 1979 & $* 2200$ & 12 & 22.0 & -73 & 20.2 & 1749.6 & 10.6 & 263 & 0.8 & 275 & 9.8 & 262 & $\mathrm{C} / \mathrm{C}$ \\
\hline 14 & 8 & 1979 & *2222 & 12 & 21.5 & -73 & 24.1 & 1753.5 & 10.5 & 262 & 0.7 & 258 & 9.8 & 262 & SATL \\
\hline 14 & 8 & 1979 & $* 2322$ & 12 & 20.0 & -73 & 34.7 & 1764.0 & 10.8 & 262 & 1.0 & 263 & 9.8 & 262 & SATL \\
\hline 14 & 8 & 1979 & *2346 & 12 & 19.4 & -73 & 39.1 & 1768.3 & 10.1 & 261 & 0.4 & 247 & 9.8 & 262 & SATL \\
\hline 15 & 8 & 1979 & ${ }^{*} 0010$ & 12 & 18.8 & -73 & 43.2 & 1772.4 & 10.7 & 261 & 0.9 & 254 & 9.8 & 262 & SATL \\
\hline 15 & 8 & 1979 & ${ }^{*} 0102$ & 12 & 17.4 & -73 & 52.6 & 1781.6 & 10.6 & 261 & 0.8 & 246 & 9.8 & 262 & SATL \\
\hline 15 & 8 & 1979 & ${ }^{*} 0134$ & 12 & 16.5 & -73 & 58.3 & 1787.3 & 11.0 & 260 & 1.3 & 246 & 9.8 & 262 & SATL \\
\hline 15 & 8 & 1979 & ${ }^{*} 0432$ & 12 & 10.9 & -74 & 31.3 & 1820.0 & 10.9 & 260 & 1.2 & 246 & 9.8 & 262 & SATL \\
\hline 15 & 8 & 1979 & ${ }^{*} 0902$ & 12 & 2.6 & -75 & 20.9 & 1869.2 & 11.0 & 262 & 1.2 & 265 & 9.8 & 262 & SATL \\
\hline 15 & 8 & 1979 & $*^{*} 1048$ & 12 & 0.0 & -75 & 40.5 & 1888.6 & 10.8 & 262 & 1.0 & 257 & 9.8 & 262 & SATL \\
\hline 15 & 8 & 1979 & $* 1230$ & 11 & 57.3 & -75 & 59.0 & 1906.9 & 10.8 & 261 & 1.0 & 255 & 9.8 & 262 & SATL \\
\hline 15 & 8 & 1979 & ${ }^{*} 1418$ & 11 & 54.4 & -76 & 18.6 & 1926.3 & 10.9 & 263 & 1.2 & 270 & 9.8 & 262 & SATL \\
\hline 15 & 8 & 1979 & ${ }^{*} 1604$ & 11 & 52.0 & -76 & 38.2 & 1945.6 & 11.0 & 263 & 1.2 & 274 & 9.8 & 262 & SATL \\
\hline 15 & 8 & 1979 & $* 1752$ & 11 & 49.7 & -76 & 58.3 & 1965.4 & 11.9 & 262 & 2.1 & 265 & 9.8 & 262 & SATL \\
\hline 15 & 8 & 1979 & 2138 & 11 & 43.8 & -77 & 43.6 & 2010.2 & 11.1 & 262 & 2.1 & 265 & 9.0 & 272 & $\mathrm{C} / \mathrm{S}$ \\
\hline 15 & 8 & 1979 & 2154 & 11 & 43.4 & -77 & 46.6 & 2013.1 & 11.9 & 262 & 2.1 & 265 & 9.8 & 262 & $\mathrm{C} / \mathrm{S}$ \\
\hline 16 & 8 & 1979 & ${ }^{*} 0014$ & 11 & 39.8 & -78 & 14.7 & 2040.8 & 12.0 & 264 & 2.2 & 274 & 9.8 & 262 & SATL \\
\hline 16 & 8 & 1979 & ${ }^{\circ} 0044$ & 11 & 39.2 & -78 & 20.8 & 2046.8 & 11.8 & 264 & 2.1 & 276 & 9.8 & 262 & SATL \\
\hline 16 & 8 & 1979 & $\bullet 0158$ & 11 & 37.8 & -78 & 35.6 & 2061.4 & 11.2 & 261 & 1.4 & 256 & 9.8 & 262 & SATL \\
\hline 16 & 8 & 1979 & 0222 & 11 & 37.1 & -78 & 40.1 & 2065.9 & 11.2 & 255 & 1.4 & 256 & 9.8 & 255 & $\mathrm{C} / \mathrm{C}$ \\
\hline 16 & 8 & 1979 & 0352 & 11 & 32.8 & -78 & 56.7 & 2082.7 & 10.5 & 200 & 1.4 & 256 & 9.8 & 193 & $\mathrm{C} / \mathrm{C}$ \\
\hline 16 & 8 & 1979 & ${ }^{*} 0510$ & 11 & 19.9 & -79 & 1.5 & 2096.4 & 10.3 & 197 & 0.9 & 251 & 9.8 & 193 & SATL \\
\hline 16 & 8 & 1979 & 0530 & 11 & 16.6 & -79 & 2.5 & 2099.9 & 10.4 & 299 & 0.9 & 251 & 9.8 & 303 & $\mathrm{C} / \mathrm{C}$ \\
\hline 16 & 8 & 1979 & 0925 & i1 & 36.4 & -79 & 38.7 & 2140.5 & 10.4 & 202 & 0.9 & 251 & 9.8 & 198 & $\mathrm{C} / \mathrm{C}$ \\
\hline 16 & 8 & 1979 & 1110 & 11 & 19.6 & -79 & 45.6 & 2158.6 & 8.9 & 070 & 0.9 & 251 & 9.8 & 070 & $\mathrm{C} / \mathrm{C}$ \\
\hline 16 & 8 & 1979 & $* 1144$ & 11 & 21.3 & -79 & 40.8 & 2163.7 & 9.3 & 070 & 0.5 & 242 & 9.8 & 070 & SATL \\
\hline 16 & 8 & 1979 & 1200 & 11 & 22.1 & -79 & 38.4 & 2166.1 & 9.3 & 066 & 0.5 & 242 & 9.8 & 066 & $\mathrm{C} / \mathrm{C}$ \\
\hline 16 & 8 & 1979 & ${ }^{*} 1214$ & 11 & 23.0 & -79 & 36.4 & 2168.3 & 8.6 & 065 & 1.2 & 255 & 9.8 & 066 & SATL \\
\hline 16 & 8 & 1979 & 1230 & 11 & 24.0 & -79 & 34.3 & 2170.6 & 6.6 & 064 & 1.2 & 255 & 7.8 & 066 & $\mathrm{C} / \mathrm{S}$ \\
\hline 16 & 8 & 1979 & 1353 & 11 & 27.9 & -79 & 25.9 & 2179.7 & 6.6 & 069 & 1.2 & 255 & 7.8 & 070 & $\mathrm{C} / \mathrm{C}$ \\
\hline 16 & 8 & 1979 & ${ }^{* 1358}$ & 11 & 28.1 & -79 & 25.4 & 2180.2 & 6.4 & 063 & 1.7 & 278 & 7.8 & 070 & SATL \\
\hline 16 & 8 & 1979 & ${ }^{*} 1425$ & 11 & 29.4 & -79 & 22.8 & 2183.1 & 7.8 & 070 & 0.0 & 044 & 7.8 & 070 & S502 \\
\hline 16 & 8 & 1979 & 1425 & 11 & 29.4 & -79 & 22.8 & 2183.1 & 0.0 & 044 & 0.0 & 044 & 0.0 & 0 & STOP \\
\hline 20 & 8 & 1979 & $* 0145$ & II & 29.5 & -79 & 22.7 & 2183.2 & 0.0 & 0 & 0.0 & 0 & 0.0 & 0 & $502 \mathrm{~A}$ \\
\hline 23 & 8 & 1979 & *2025 & 11 & 29.5 & -79 & 22.7 & 2183.2 & 0.0 & 0 & 0.0 & 0 & 0.0 & 0 & $502 \mathrm{~B}$ \\
\hline 25 & 8 & 1979 & •1550 & 11 & 29.5 & -79 & 22.7 & 2183.2 & 0.0 & 0 & 0.0 & 0 & 0.0 & 0 & $502 \mathrm{C}$ \\
\hline 27 & 8 & 1979 & •2208 & 11 & 29.5 & -79 & 22.7 & 2183.2 & 1.1 & 216 & 1.1 & 216 & 0.0 & 0 & DEP \\
\hline 27 & 8 & 1979 & 2208 & 11 & 29.5 & -79 & 22.7 & 2183.2 & 10.2 & 194 & 1.1 & 216 & 9.2 & 191 & $\mathrm{U} / \mathrm{W}$ \\
\hline 27 & 8 & 1979 & 2222 & 11 & 27.2 & -79 & 23.3 & 2185.6 & 10.6 & 194 & 1.1 & 216 & 9.6 & 191 & $\mathrm{C} / \mathrm{S}$ \\
\hline 27 & 8 & 1979 & $\cdot 2348$ & 11 & 12.4 & -79 & 26.9 & 2200.8 & 10.6 & 193 & 1.0 & 212 & 9.6 & 191 & SATL \\
\hline 28 & 8 & 1979 & •0134 & 10 & 54.2 & -79 & 31.2 & 2219.5 & 8.4 & 192 & 1.2 & 002 & 9.6 & 191 & SATL \\
\hline 28 & 8 & 1979 & ${ }^{*} 0446$ & 10 & 28.0 & -79 & 37.0 & 2246.3 & 10.9 & 190 & 1.3 & 184 & 9.6 & 191 & STOP \\
\hline 28 & 8 & 1979 & 0446 & 10 & 28.0 & -79 & 37.0 & 2246.3 & 1.3 & 184 & 1.3 & 184 & 0.0 & 0 & STOP \\
\hline 3 & 9 & 1979 & $\bullet 0012$ & 7 & 26.4 & -79 & 50.1 & 2428.4 & 0.7 & 163 & 0.7 & 163 & 0.0 & 0 & DEP \\
\hline 3 & 9 & 1979 & 0015 & 7 & 26.4 & -79 & 50.1 & 2428.4 & 9.5 & 239 & 0.7 & 163 & 9.4 & 243 & $\mathrm{U} / \mathrm{W}$ \\
\hline 3 & 9 & 1979 & 0030 & 7 & 25.1 & -79 & 52.2 & 2430.8 & 9.7 & 221 & 0.7 & 163 & 9.4 & 224 & $\mathrm{C} / \mathrm{C}$ \\
\hline 3 & 9 & 1979 & 0120 & 7 & 19.0 & -79 & 57.5 & 2438.9 & 5.2 & 236 & 0.7 & 163 & 5.0 & 243 & $\mathrm{C} / \mathrm{C}$ \\
\hline 3 & 9 & 1979 & 0135 & 7 & 18.2 & -79 & 58.5 & 2440.2 & 9.1 & 239 & 0.7 & 163 & 9.0 & 243 & $\mathrm{C} / \mathrm{S}$ \\
\hline 3 & 9 & 1979 & ${ }^{*} 0234$ & 7 & 13.6 & -80 & 6.3 & 2449.2 & 9.1 & 242 & 0.2 & 187 & 9.0 & 243 & SATL \\
\hline 3 & 9 & 1979 & ${ }^{*} 0422$ & 7 & 5.8 & -80 & 20.9 & 2465.6 & 9.5 & 243 & 0.6 & 235 & 9.0 & 243 & SATL \\
\hline 3 & 9 & 1979 & 0430 & 7 & 5,2 & -80 & 22.0 & 2466.9 & 9.5 & 258 & 0.6 & 235 & 9.0 & 259 & $\mathrm{C} / \mathrm{C}$ \\
\hline 3 & 9 & 1979 & ${ }^{*} 0838$ & 6 & 56.8 & -81 & 0.7 & 2506.2 & 9.6 & 256 & 0.7 & 224 & 9.0 & 259 & SATL \\
\hline 3 & 9 & 1979 & $* 1118$ & 6 & 50.8 & -81 & 25.8 & 2531.8 & 9.4 & 256 & 0.7 & 210 & 9.0 & 259 & SATL \\
\hline 3 & 9 & 1979 & • 1332 & 6 & 45.7 & -81 & 46.4 & 2552.9 & 10.2 & 257 & 1.2 & 246 & 9.0 & 259 & SATL \\
\hline 3 & 9 & 1979 & *2018 & 6 & 30.8 & -82 & 53.9 & 2621.6 & 10.4 & 262 & 1.5 & 278 & 9.0 & 259 & SATL \\
\hline 3 & 9 & 1979 & 2105 & 6 & 29.6 & -83 & 2.0 & 2629.7 & 10.4 & 263 & 1.5 & 278 & 9.0 & 261 & $\mathrm{C} / \mathrm{C}$ \\
\hline 3 & 9 & 1979 & $* 2140$ & 6 & 28.9 & -83 & 8.1 & 2635.8 & 9.9 & 260 & 0.9 & 255 & 9.0 & 261 & SATL \\
\hline 3 & 9 & 1979 & *2202 & 6 & 28.3 & -83 & 11.7 & 2639.4 & 9.9 & 261 & 0.9 & 265 & 9.0 & 261 & SATL \\
\hline 3 & 9 & 1979 & 2310 & 6 & 26.6 & -83 & 22.8 & 2650.6 & 9.9 & 270 & 0.9 & 265 & 9.0 & 270 & $\mathrm{C} / \mathrm{C}$ \\
\hline 3 & 9 & 1979 & $\cdot 2322$ & 6 & 26.6 & -83 & 24.8 & 2652.6 & 9.9 & 269 & 0.9 & 258 & 9.0 & 270 & SATL \\
\hline 3 & 9 & 1979 & 2330 & 6 & 26.6 & -83 & 26.1 & 2653.9 & 9.9 & 260 & 0.9 & 258 & 9.0 & 260 & $\mathrm{C} / \mathrm{C}$ \\
\hline 4 & 9 & 1979 & ${ }^{*} 1216$ & 6 & 4.1 & -85 & 31.7 & 2780.7 & 10.0 & 263 & 1.1 & 286 & 9.0 & 260 & SATL \\
\hline
\end{tabular}




\section{J. V. GARDNER}

Table 1. (Continued).

\begin{tabular}{|c|c|c|c|c|c|c|c|c|c|c|c|c|c|c|c|}
\hline \multirow[b]{2}{*}{ Day } & \multirow[b]{2}{*}{ Month } & \multirow[b]{2}{*}{ Year } & & Lati & ude & Long & tude & & & tual & & rift & Dead R & eckoning & \\
\hline & & & Time & Degree & Minute & Degree & Minute & Distance , & Speed & Course & Speed & Heading & Speed & Course & Comments $\mathrm{a}$ \\
\hline 4 & 9 & 1979 & $* 1240$ & 6 & 3.6 & -85 & 35.7 & 2784.7 & 9.8 & 261 & 0.9 & 274 & 9.0 & 260 & SATL \\
\hline 4 & 9 & 1979 & ${ }^{*} 1404$ & 6 & 1.5 & -85 & 49.4 & 2798.5 & 10.0 & 265 & 1.3 & 300 & 9.0 & 260 & SATL \\
\hline 4 & 9 & 1979 & •1444 & 6 & 0.9 & -85 & 56.1 & 2805.2 & 9.6 & 261 & 0.6 & 279 & 9.0 & 260 & SATL \\
\hline 4 & 9 & 1979 & $\bullet$ & 5 & 58.4 & -86 & 12.3 & 2821.5 & 9.6 & 259 & 0.6 & 250 & 9.0 & 260 & SATL \\
\hline 4 & 9 & 1979 & $* 2112$ & 5 & 49.9 & -86 & 57.6 & 2867.4 & 9.8 & 260 & 0.8 & 264 & 9.0 & 260 & SATL \\
\hline 4 & 9 & 1979 & 2215 & 5 & 48.2 & -87 & 7.9 & 2877.7 & 9.8 & 259 & 0.8 & 264 & 9.0 & 259 & $\mathrm{C} / \mathrm{C}$ \\
\hline 4 & 9 & 1979 & *2354 & 5 & 45.2 & -87 & 23.9 & 2894.0 & 9.8 & 258 & 0.8 & 247 & 9.0 & 259 & SATL \\
\hline 5 & 9 & 1979 & •0140 & 5 & 41.6 & -87 & 40.9 & 2911.3 & 9.8 & 257 & 0.9 & 231 & 9.0 & 259 & SATL \\
\hline 5 & 9 & 1979 & $* 0350$ & 5 & 36.7 & -88 & 1.6 & 2932.4 & 10.0 & 257 & 1.0 & 240 & 9.0 & 259 & SATL \\
\hline 5 & 9 & 1979 & •1028 & 5 & 21.8 & -89 & 6.5 & 2998.7 & 10.0 & 259 & 1.0 & 260 & 9.0 & 259 & SATL \\
\hline 5 & 9 & 1979 & $\bullet 1128$ & 5 & 19.9 & -89 & 16.4 & 3008.8 & 10.1 & 259 & 1.1 & 256 & 9.0 & 259 & SATL \\
\hline 5 & 9 & 1979 & $* 1152$ & 5 & 19.1 & -89 & 20.4 & 3012.8 & 9.9 & 259 & 0.9 & 263 & 9.0 & 259 & SATL \\
\hline 5 & 9 & 1979 & •1314 & 5 & 16.6 & -89 & 33.8 & 3026.4 & 10.6 & 256 & 1.7 & 241 & 9.0 & 259 & SATL \\
\hline 5 & 9 & 1979 & ${ }^{*} 1340$ & 5 & 15.5 & -89 & 38.3 & 3031.0 & 10.2 & 260 & 1.2 & 272 & 9.0 & 259 & SATL \\
\hline 5 & 9 & 1979 & • 1520 & 5 & 12.7 & -89 & 55.1 & 3048.0 & 9.8 & 259 & 0.9 & 253 & 9.0 & 259 & SATL \\
\hline 5 & 9 & 1979 & $* 1704$ & 5 & 9.3 & -90 & 11.9 & 3065.1 & 9.8 & 257 & 0.8 & 240 & 9.0 & 259 & SATL \\
\hline 5 & 9 & 1979 & *2136 & 4 & 59.6 & -90 & 55.4 & 3109.5 & 9.7 & 258 & 0.8 & 241 & 9.0 & 259 & SATL \\
\hline 6 & 9 & 1979 & ${ }^{*} 0938$ & 4 & 34.5 & -92 & 50.2 & 3226.6 & 10.0 & 258 & 1.0 & 248 & 9.0 & 259 & SATL \\
\hline 6 & 9 & 1979 & *1056 & 4 & 31.8 & -93 & 2.9 & 3239.5 & 8.7 & 254 & 0.8 & 142 & 9.0 & 259 & SATL \\
\hline 6 & 9 & 1979 & •1124 & 4 & 30.7 & -93 & 6.8 & 3243.6 & 9.6 & 258 & 0.7 & 247 & 9.0 & 259 & SATL \\
\hline 6 & 9 & 1979 & *1228 & 4 & 28.6 & -93 & 16.9 & 3253.9 & 9.5 & 257 & 0.6 & 221 & 9.0 & 259 & SATL \\
\hline 6 & 9 & 1979 & $* 1250$ & 4 & 27.8 & -93 & 20.3 & 3257.3 & 9.7 & 257 & 0.8 & 232 & 9.0 & 259 & SATL \\
\hline 6 & 9 & 1979 & •1414 & 4 & 24.7 & -93 & 33.6 & 3271.0 & 9.7 & 258 & 0.7 & 242 & 9.0 & 259 & SATL \\
\hline 6 & 9 & 1979 & 1500 & 4 & 23.1 & -93 & 40.9 & 3278.4 & 9.7 & 261 & 0.7 & 242 & 9.0 & 262 & $\mathrm{C} / \mathrm{C}$ \\
\hline 6 & 9 & 1979 & • 1558 & 4 & 21.6 & -93 & 50.1 & 3287.7 & 9.6 & 261 & 0.6 & 241 & 9.0 & 262 & SATL \\
\hline 6 & 9 & 1979 & • 2114 & 4 & 13.5 & -94 & 39.9 & 3338.0 & 9.9 & 262 & 0.9 & 265 & 9.0 & 262 & SATL \\
\hline 6 & 9 & 1979 & $* 2226$ & 4 & 11.9 & -94 & 51.7 & 3349.9 & 9.7 & 264 & 0.8 & 284 & 9.0 & 262 & SATL \\
\hline 6 & 9 & 1979 & *2300 & 4 & 11.3 & -94 & 57.2 & 3355.4 & 9.7 & 262 & 0.7 & 265 & 9.0 & 262 & SATL \\
\hline 6 & 9 & 1979 & 2325 & 4 & 10.8 & -95 & 1.2 & 3359.5 & 9.7 & 261 & 0.7 & 265 & 9.0 & 261 & $\mathrm{C} / \mathrm{C}$ \\
\hline 7 & 9 & 1979 & $\bullet 0004$ & 4 & 9.8 & -95 & 7.5 & 3365.8 & 9.6 & 261 & 0.6 & 261 & 9.0 & 261 & SATL \\
\hline 7 & 9 & 1979. & *0028 & 4 & 9.2 & -95 & 11.3 & 3369.6 & 9.4 & 260 & 0.4 & 235 & 9.0 & 261 & SATL \\
\hline 7 & 9 & 1979 & 0045 & 4 & 8.7 & -95 & 13.9 & 3372.3 & 9.4 & 259 & 0.4 & 235 & 9.0 & 260 & $\mathrm{C} / \mathrm{C}$ \\
\hline 7 & 9 & 1979 & $* 0150$ & 4 & 6.8 & -95 & 23.9 & 3382.4 & 10.1 & 259 & 1.1 & 255 & 9.0 & 260 & SATL \\
\hline 7 & 9 & 1979 & ${ }^{*} 0216$ & 4 & 6.0 & -95 & 28.2 & 3386.8 & 9.8 & 258 & 0.9 & 232 & 9.0 & 260 & SATL \\
\hline 7 & 9 & 1979 & 0305 & 4 & 4.3 & -95 & 36.0 & 3394.8 & 5.8 & 256 & 0.9 & 232 & 5.0 & 260 & $\mathrm{C} / \mathrm{S}$ \\
\hline 7 & 9 & 1979 & *0322 & 4 & 3.9 & -95 & 37.6 & 3396.4 & 3.6 & 279 & 2.0 & 042 & 5.0 & 260 & SATL \\
\hline 7 & 9 & 1979 & ${ }^{*} 0322$ & 4 & 4.0 & -95 & 38.2 & 3397.0 & 5.0 & 260 & 0.0 & 0 & 5.0 & 260 & S503 \\
\hline 7 & 9 & 1979 & 0322 & 4 & 4.0 & -95 & 38.2 & 3397.0 & 0.0 & 0 & 0.0 & 0 & 0.0 & 0 & STOP \\
\hline 13 & 9 & 1979 & •1704 & 4 & 4.0 & -95 & 38.2 & 3397.0 & 0.8 & 079 & 0.8 & 079 & 0.0 & 0 & DEP \\
\hline 13 & 9 & 1979 & 1704 & 4 & 4.0 & -95 & 38.2 & 3397.0 & 9.7 & 098 & 0.8 & 079 & 9.0 & 100 & $\mathrm{U} / \mathrm{W}$ \\
\hline 13 & 9 & 1979 & 1707 & 4 & 3.9 & -95 & 37.7 & 3397.5 & 9.6 & 110 & 0.8 & 079 & 9.0 & 113 & $\mathrm{C} / \mathrm{C}$ \\
\hline 13 & 9 & 1979 & • 2032 & 3 & 52.4 & -95 & 6.8 & 3430.4 & 9.7 & 110 & 0.8 & 078 & 9.0 & 113 & SATL \\
\hline 13 & 9 & 1979 & *2216 & 3 & 46.6 & -94 & 51.0 & 3447.2 & 9.4 & 110 & 0.6 & 065 & 9.0 & 113 & SATL \\
\hline 13 & 9 & 1979 & *2304 & 3 & 44.0 & -94 & 43.9 & 3454.8 & 9.5 & 110 & 0.7 & 070 & 9.0 & 113 & SATL \\
\hline 13 & 9 & 1979 & 2310 & 3 & 43.7 & -94 & 43.0 & 3455.7 & 9.5 & 112 & 0.7 & 070 & 9.0 & 115 & $\mathrm{C} / \mathrm{C}$ \\
\hline 14 & 9 & 1979 & ${ }^{*} 0420$ & 3 & 25.3 & -93 & 57.3 & 3504.9 & 9.6 & 114 & 0.7 & 097 & 9.0 & 115 & SATL \\
\hline 14 & 9 & 1979 & •0948 & 3 & 4.1 & -93 & 9.1 & 3557.5 & 9.4 & 117 & 0.5 & 149 & 9.0 & 115 & SATL \\
\hline 14 & 9 & 1979 & ${ }^{*} 1038$ & 3 & 0.6 & -93 & 2.1 & 3565.3 & 9.3 & 114 & 0.4 & 087 & 9.0 & 115 & SATL \\
\hline 14 & 9 & 1979 & *1116 & 2 & 58.2 & -92 & 56.7 & 3571.2 & 9.9 & 114 & 0.9 & 109 & 9.0 & 115 & SATL \\
\hline 14 & 9 & 1979 & •1138 & 2 & 56.7 & -92 & 53.4 & 354.8 & 9.6 & 114 & 0.6 & 101 & 9.0 & 115 & SATL \\
\hline 14 & 9 & 1979 & *1302 & 2 & 51.2 & -92 & 41.1 & 3588.3 & 9.6 & 115 & 0.6 & 109 & 9.0 & 115 & SATL \\
\hline 14 & 9 & 1979 & *1326 & 2 & 49.6 & -92 & 37.6 & 3592.1 & 9.7 & 114 & 0.7 & 105 & 9.0 & 115 & SATL \\
\hline 14 & 9 & 1979 & 1700 & 2 & 35.4 & -92 & 6.0 & 3626.7 & 7.7 & 114 & 0.7 & 105 & 7.0 & 115 & $\mathrm{C} / \mathrm{S}$ \\
\hline 14 & 9 & 1979 & *2314 & 2 & 15.8 & -91 & 22.1 & 3674.8 & 7.4 & 113 & 0.5 & 087 & 7.0 & 115 & SATL \\
\hline 15 & 9 & 1979 & ${ }^{*} 0038$ & 2 & 11.7 & -91 & 12.5 & 3685.2 & 7.0 & 113 & 0.3 & 035 & 7.0 & 115 & SATL \\
\hline 15 & 9 & 1979 & ${ }^{*} 0102$ & 2 & 10.6 & -91 & 9.9 & 3688.0 & 7.1 & 110 & 0.6 & 032 & 7.0 & 115 & SATL \\
\hline 15 & 9 & 1979 & 0105 & 2 & 10.5 & -91 & 9.6 & 3688.4 & 9.1 & 111 & 0.6 & 032 & 9.0 & 115 & $\mathrm{C} / \mathrm{S}$ \\
\hline 15 & 9 & 1979 & •0310 & 2 & 3.6 & -90 & 51.9 & 3707.3 & 9.4 & 111 & 0.8 & 051 & 9.0 & 115 & SATL \\
\hline 15 & 9 & 1979 & 0816 & 1 & 46.7 & -90 & 7.2 & 3755.1 & 7.4 & 110 & 0.8 & $05 i$ & 7.0 & 115 & $\mathrm{C} / \mathrm{S}$ \\
\hline 15 & 9 & 1979 & *0856 & 1 & 45.0 & -90 & 2.6 & 3760.0 & 7.1 & 110 & 0.6 & 032 & 7.0 & 115 & SATL \\
\hline 15 & 9 & 1979 & *0942 & 1 & 43.1 & -89 & 57.5 & 3765.4 & 7.8 & 110 & 1.1 & 074 & 7.0 & 115 & SATL \\
\hline 15 & 9 & 1979 & $* 1130$ & 1 & 38.3 & -89 & 44.2 & 3779.6 & 8.0 & 109 & 1.3 & 073 & 7.0 & 115 & SATL \\
\hline 15 & 9 & 1979 & *1212 & 1 & 36.5 & -89 & 38.9 & 3785.2 & 6.6 & 115 & 0.4 & 301 & 7.0 & 115 & SATL \\
\hline 15 & 9 & 1979 & •1236 & 1 & 35.4 & -89 & 36.5 & 3787.8 & 7.9 & 113 & 1.0 & 095 & 7.0 & 115 & SATL \\
\hline 15 & 9 & 1979 & ${ }^{*} 1438$ & 1 & 29.2 & -89 & 21.6 & 3803.9 & 7.8 & 113 & 0.8 & 093 & 7.0 & 115 & SATL \\
\hline 15 & 9 & 1979 & •1624 & 1 & 23.9 & -89 & 8.9 & 3817.7 & 7.4 & 113 & 0.5 & 083 & 7.0 & 115 & SATL \\
\hline 15 & 9 & 1979 & *2032 & 1 & 11.9 & -88 & 40.8 & 3848.2 & 7.5 & 110 & 0.8 & 060 & 7.0 & 115 & SATL \\
\hline 15 & 9 & 1979 & *2114 & 1 & 10.1 & -88 & 35.9 & 3853.4 & 7.1 & 112 & 0.4 & 042 & 7.0 & 115 & SATL \\
\hline 15 & 9 & 1979 & $* 2220$ & 1 & 7.2 & -88 & 28.6 & 3861.3 & 7.1 & 112 & 0.4 & 044 & 7.0 & 115 & SATL \\
\hline 15 & 9 & 1979 & *2308 & 1 & 5.4 & -88 & 24.2 & 3866.1 & 7.4 & 115 & 0.4 & 112 & 7.0 & 115 & SATL \\
\hline 15 & 9 & 1979 & *2348 & 1 & 2.9 & -88 & 18.8 & 3872.0 & 7.0 & 113 & 0.2 & 034 & 7.0 & 115 & SATL \\
\hline 16 & 9 & 1979 & ${ }^{*} 0014$ & 1 & 1.7 & -88 & 16.0 & 3875.1 & 7.2 & 116 & 0.2 & 139 & 7.0 & 115 & SATL \\
\hline 16 & 9 & 1979 & ${ }^{*} 0136$ & 0 & 57.4 & -88 & 7.1 & 3884.9 & 7.1 & 113 & 0.2 & 048 & 7.0 & 115 & SATL \\
\hline 16 & 9 & 1979 & $* 0348$ & 0 & 51.2 & -87 & 52.8 & 3900.5 & 6.6 & 116 & 0.4 & 281 & 7.0 & 115 & SATL \\
\hline
\end{tabular}


Table 1. (Continued).

\begin{tabular}{|c|c|c|c|c|c|c|c|c|c|c|c|c|c|c|c|}
\hline \multirow[b]{2}{*}{ Day } & \multirow[b]{2}{*}{ Month } & \multirow[b]{2}{*}{ Year } & \multirow[b]{2}{*}{ Time } & \multicolumn{2}{|c|}{ Latitude } & \multicolumn{2}{|c|}{ Longitude } & \multirow[b]{2}{*}{ Distance } & \multicolumn{2}{|c|}{ Actual } & \multicolumn{2}{|c|}{ Drift } & \multicolumn{2}{|c|}{ Dead Reckoning } & \multirow[b]{2}{*}{ Comments $^{\mathrm{a}}$} \\
\hline & & & & Degree & Minute & Degree & Minute & & Speed & Course & Speed & Heading & Speed & Course & \\
\hline 16 & 9 & 1979 & 0528 & 0 & 46.4 & -87 & 42.9 & 3911.5 & 8.6 & 116 & 0.4 & 281 & 9.0 & 115 & $\mathrm{C} / \mathrm{S}$ \\
\hline 16 & 9 & 1979 & •0806 & 0 & 36.6 & -87 & 22.5 & 3934.1 & 8.4 & 114 & 0.6 & 310 & 9.0 & 115 & SATL \\
\hline 16 & 9 & 1979 & *0848 & 0 & 34.2 & -87 & 17.1 & 3940.1 & 8.3 & 116 & 0.7 & 283 & 9.0 & 115 & SATL \\
\hline 16 & 9 & 1979 & *0952 & 0 & 30.3 & -87 & 9.1 & 3949.0 & 8.2 & 117 & 0.8 & 274 & 9.0 & 115 & SATL \\
\hline 16 & 9 & 1979 & $* 1032$ & 0 & 27.8 & -87 & 4.2 & 3954.5 & 8.6 & 116 & 0.4 & 285 & 9.0 & 115 & SATL \\
\hline 16 & 9 & 1979 & $* 1124$ & 0 & 24.6 & -86 & 57.5 & 3961.9 & 8.1 & 116 & 0.9 & 290 & 9.0 & 115 & SATL \\
\hline 16 & 9 & 1979 & ${ }^{*} 1200$ & 0 & 22.5 & -86 & 53.1 & 3966.8 & 8.1 & 117 & 1.0 & 282 & 9.0 & 115 & SATL \\
\hline 16 & 9 & 1979 & $* 1310$ & 0 & 18.3 & -86 & 44.7 & 3976.1 & 8.3 & 116 & 0.7 & 278 & 9.0 & 115 & SATL \\
\hline 16 & 9 & 1979 & •2126 & 0 & -12.5 & -85 & 42.9 & 4045.2 & 8.5 & 115 & 0.5 & 292 & 9.0 & 115 & SATL \\
\hline 16 & 9 & 1979 & *2204 & 0 & -14.8 & -85 & 38.0 & 4050.6 & 8.4 & 115 & 0.6 & 296 & 9.0 & 115 & SATL \\
\hline 16 & 9 & 1979 & $* 2300$ & 0 & -18.1 & -85 & 30.9 & 4058.4 & 8.6 & 117 & 0.5 & 265 & 9.0 & 115 & SATL \\
\hline 17 & 9 & 1979 & $* 0046$ & 0 & -24.9 & -85 & 17.4 & 4073.5 & 8.5 & 117 & 0.6 & 262 & 9.0 & 115 & SATL \\
\hline 17 & 9 & 1979 & 0242 & 0 & -32.4 & -85 & 2.7 & 4090.0 & 8.8 & 115 & 0.2 & 286 & 9.0 & 115 & SATL \\
\hline 17 & 9 & 1979 & $* 0428$ & 0 & -39.0 & -84 & 48.7 & 4105.5 & 8.7 & 117 & 0.5 & 252 & 9.0 & 115 & SATL \\
\hline 17 & 9 & 1979 & 0900 & 0 & -56.9 & -84 & 13.7 & 4144.8 & 8.8 & 113 & 0.3 & 354 & 9.0 & 115 & SATL \\
\hline 17 & 9 & 1979 & $* 1036$ & -1 & 2.4 & -84 & 0.7 & 4159.0 & 8.9 & 113 & 0.3 & 008 & 9.0 & 115 & SATL \\
\hline 17 & 9 & 1979 & $* 1058$ & -1 & 3.7 & -83 & 57.7 & 4162.2 & 8.9 & 111 & 0.6 & 012 & 9.0 & 115 & SATL \\
\hline 17 & 9 & 1979 & $* 1222$ & -1 & 8.2 & -83 & 46.1 & 4174.7 & 8.7 & 114 & 0.3 & 331 & 9.0 & 115 & SATL \\
\hline 17 & 9 & 1979 & $* 1246$ & -1 & 9.6 & -83 & 42.9 & 4178.2 & 8.8 & 110 & 0.7 & 009 & 9.0 & 115 & SATL \\
\hline 17 & 9 & 1979 & $* 1408$ & -1 & 13.8 & -83 & 31.6 & 4190.2 & 8.7 & 111 & 0.7 & 355 & 9.0 & 115 & SATL \\
\hline 17 & 9 & 1979 & *1554 & -1 & 19.3 & -83 & 17.3 & 4205.5 & 9.7 & 113 & 0.7 & 089 & 9.0 & 115 & SATL \\
\hline 17 & 9 & 1979 & 1745 & -1 & 26.3 & -83 & 0.9 & 4223.4 & 5.7 & 112 & 0.7 & 089 & 5.0 & 115 & $\mathrm{C} / \mathrm{S}$ \\
\hline 17 & 9 & 1979 & $* 2110$ & -1 & 33.5 & -82 & 42.9 & 4242.7 & 5.9 & 110 & 1.0 & 085 & 5.0 & 115 & SATL \\
\hline 17 & 9 & 1979 & *2212 & -1 & 35.6 & -82 & 37.2 & 4248.8 & 6.6 & 112 & 1.6 & 103 & 5.0 & 115 & SATL \\
\hline 17 & 9 & 1979 & 2220 & -1 & 35.9 & -82 & 36.4 & 4249.7 & 3.1 & 109 & 1.6 & 103 & 1.5 & 115 & $\mathrm{C} / \mathrm{S}$ \\
\hline 17 & 9 & 1979 & 2244 & -1 & 36.3 & -82 & 35.2 & 4250.9 & 6.6 & 112 & 1.6 & 103 & 5.0 & 115 & $\mathrm{C} / \mathrm{S}$ \\
\hline 17 & 9 & 1979 & $* 2356$ & -1 & 39.3 & -82 & 27.9 & 4258.8 & 6.5 & 113 & 1.5 & 105 & 5.0 & 115 & SATL \\
\hline 18 & 9 & 1979 & $* 0020$ & -1 & 40.3 & -82 & 25.5 & 4261.4 & 6.0 & 112 & 1.0 & 095 & 5.0 & 115 & SATL \\
\hline 18 & 9 & 1979 & 0048 & -1 & 41.3 & -82 & 22.9 & 4264.2 & 7.0 & 109 & 1.0 & 095 & 6.0 & 112 & $\mathrm{C} / \mathrm{S}$ \\
\hline 18 & 9 & 1979 & 0105 & -1 & 42.0 & -82 & 21.0 & 4266.2 & 10.0 & 110 & 1.0 & 095 & 9.0 & 112 & $\mathrm{C} / \mathrm{S}$ \\
\hline 18 & 9 & 1979 & •0810 & -2 & 6.5 & -81 & 14.5 & 4337.0 & 9.5 & 105 & 1.3 & 042 & 9.0 & 112 & SATL \\
\hline 18 & 9 & 1979 & 0828 & -2 & 7.2 & -81 & 11.7 & 4339.9 & 9.7 & 096 & 1.3 & 042 & 9.0 & 102 & $\mathrm{C} / \mathrm{C}$ \\
\hline 18 & 9 & 1979 & *0845 & -2 & 7.55 & -81 & 9.04 & 4342.7 & 9.7 & 096 & 0.0 & 0 & 9.0 & 102 & DR \\
\hline
\end{tabular}

a Symbols for comments: $\mathrm{DR}=$ dead reckoning; $\mathrm{C} / \mathrm{S}=$ change speed; $\mathrm{SATL}=$ satellite $\mathrm{C} / \mathrm{C}=$ course change; $\mathrm{STOP}=$ stop; $\mathrm{U} / \mathrm{W}=$ underway; $\mathrm{DEP}=$ depart; PORT = port; $*=$ satellite fix.

off because of ship traffic before we completed the traverse up the inner trench wall.

\section{REFERENCES}

Chase, T. E., Menard, H. W., and Mammerickx, J., 1970. Bathymetry of the North Pacific. Scripps Inst. Ocean., IMR Tech. Rept., 15.

Edgar, N. T., Saunders, J. B., et al., 1973. Init. Repts. DSDP, 15: Washington (U.S. Govt. Printing Office).

Hays, J. D., et al., 1972. Init. Repts. DSDP, 9: Washington (U.S. Govt. Printing Office).
Holcombe, T. L., 1975. Caribbean bathymetry and sediments. Geology, Geophysics, and Resources of the Caribbean. Report of IDOE Workshop on Geology and Marine Geophysics of the Caribbean Region and Its Resources, Kingston, Jamaica, pp. 27-62.

Mammerickx, J., Smith, S. M., Taylor, I. L., and Chase, T. E., 1974. Bathymetry of the South Pacific. Scripps Inst. Oceanog., IMR Tech. Rept., 48A.

van Andel, Tj. H., Heath, G. R., and Moore, T. C., Jr., 1975. Cenozoic history and paleooceanography of the central equatorial $\mathrm{Pa}$ cific Ocean. Geol. Soc. Am. Mem., 143. 

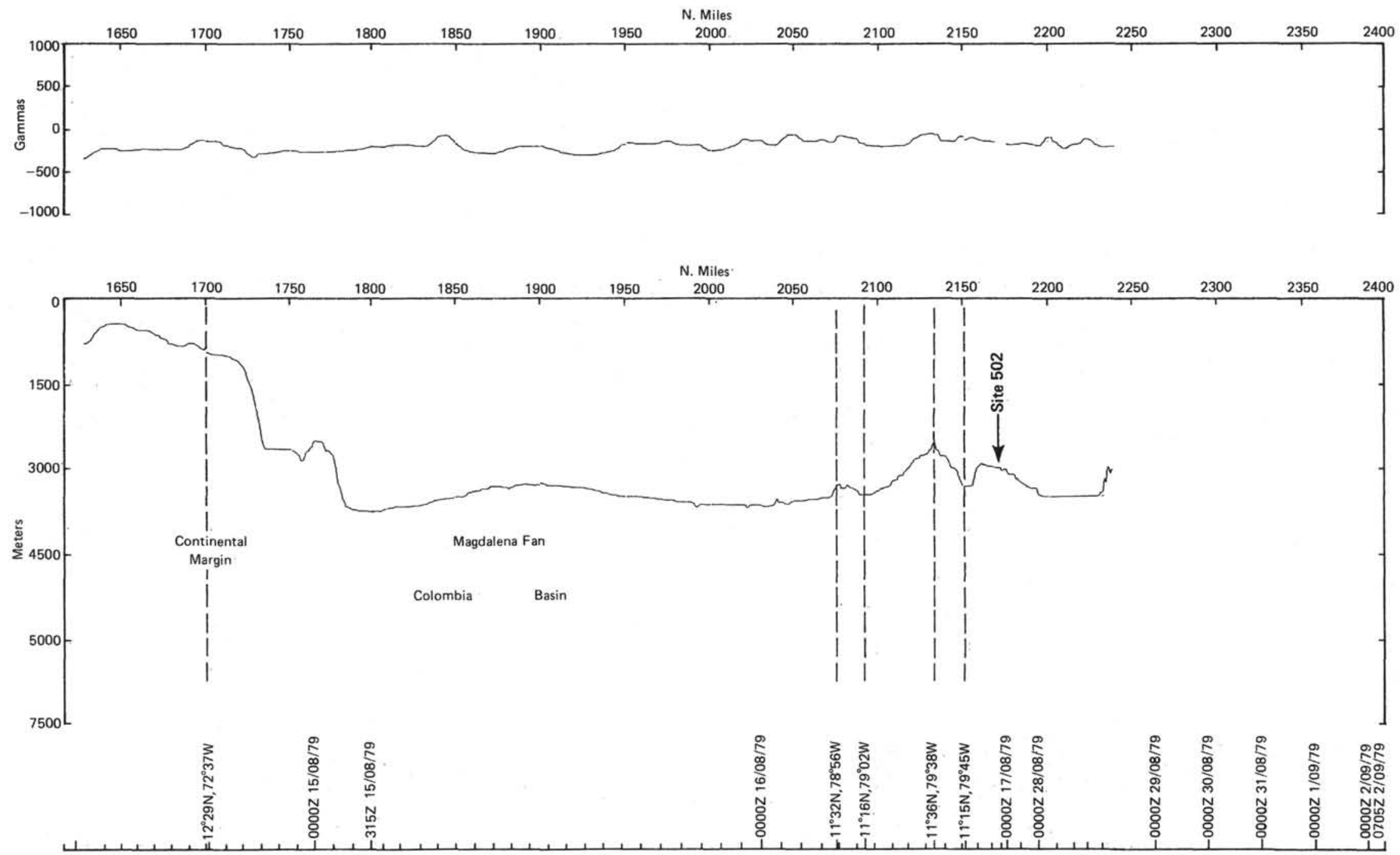

Figure 2. Profiles of magnetic anomalies (upper) and bathymetry (lower) for Leg 68. 

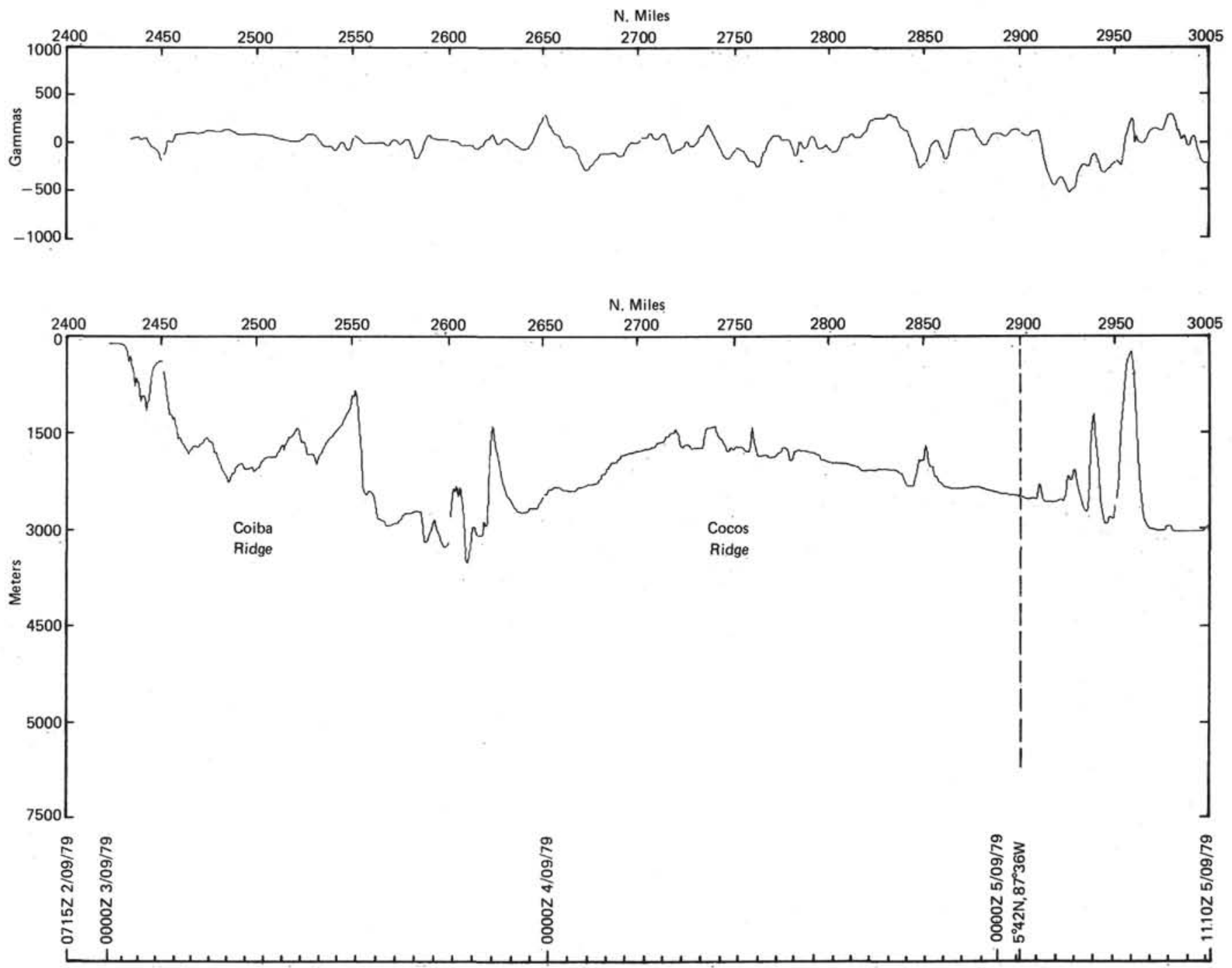

Figure 3. Profiles of magnetic anomalies (upper) and bathymetry (lower) for Leg 68 . 

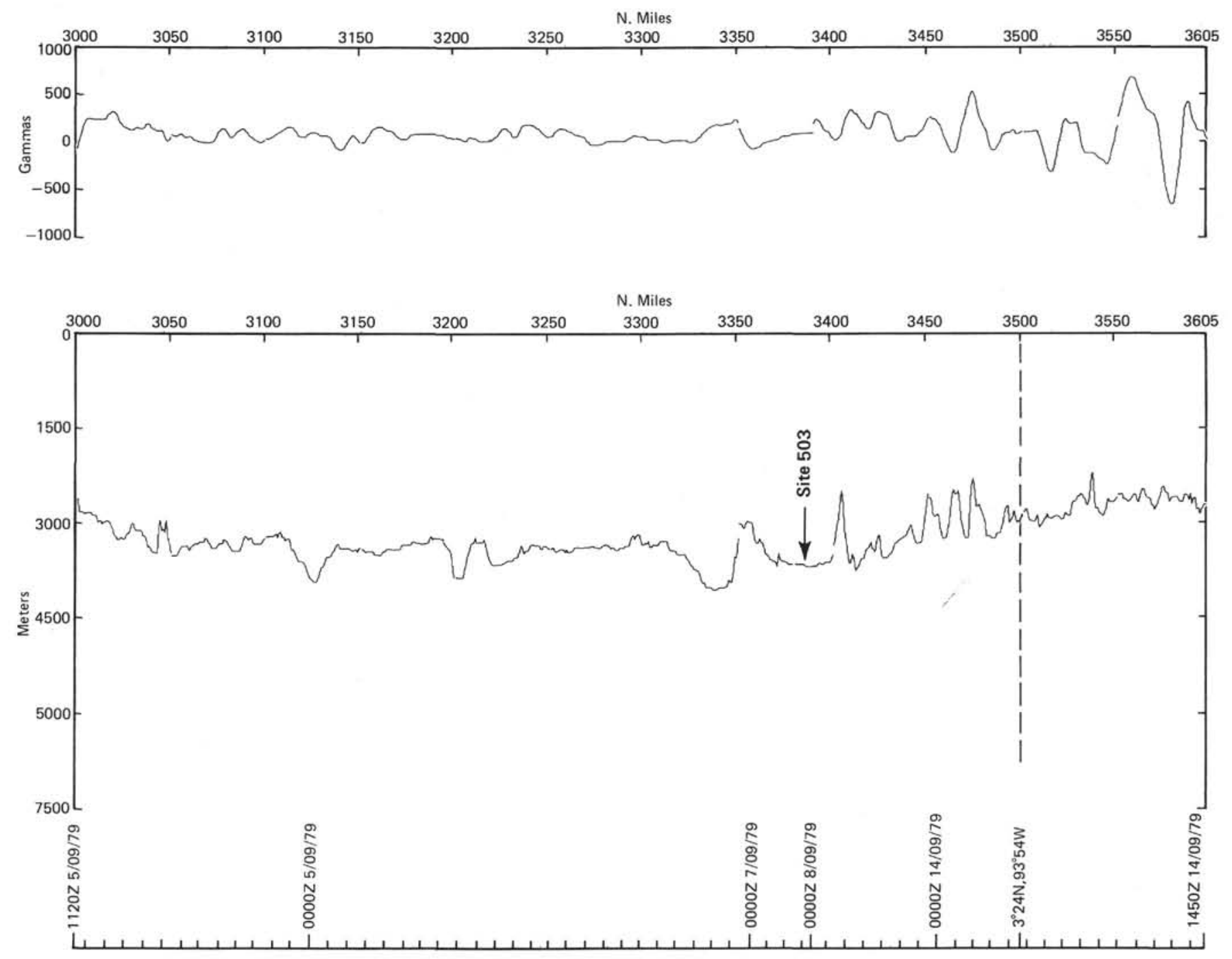

Figure 4. Profiles of magnetic anomalies (upper) and bathymetry (lower) for Leg 68 . 

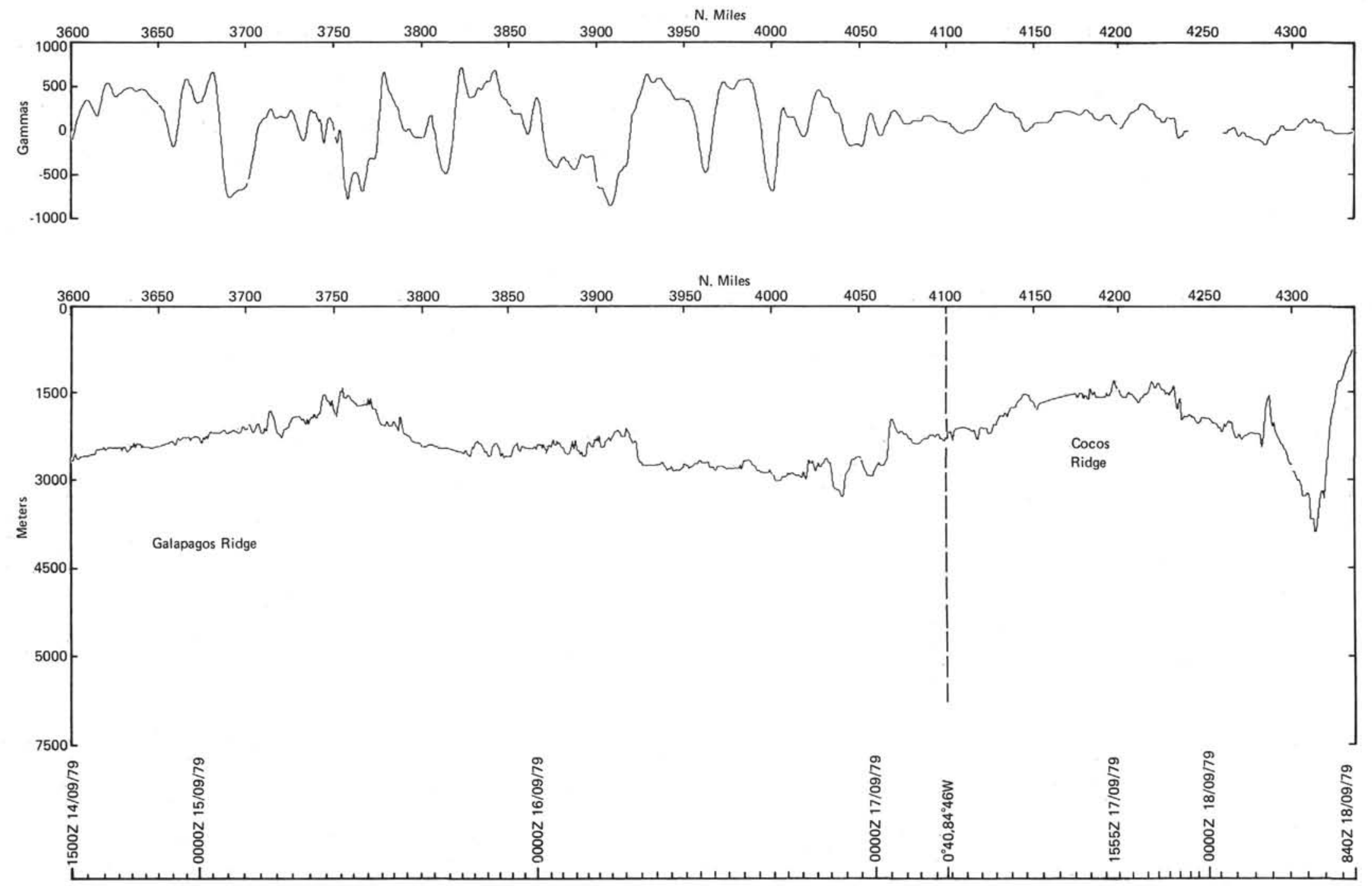

Figure 5. Profiles of magnetic anomalies (upper) and bathymetry (lower) for Leg 68. 


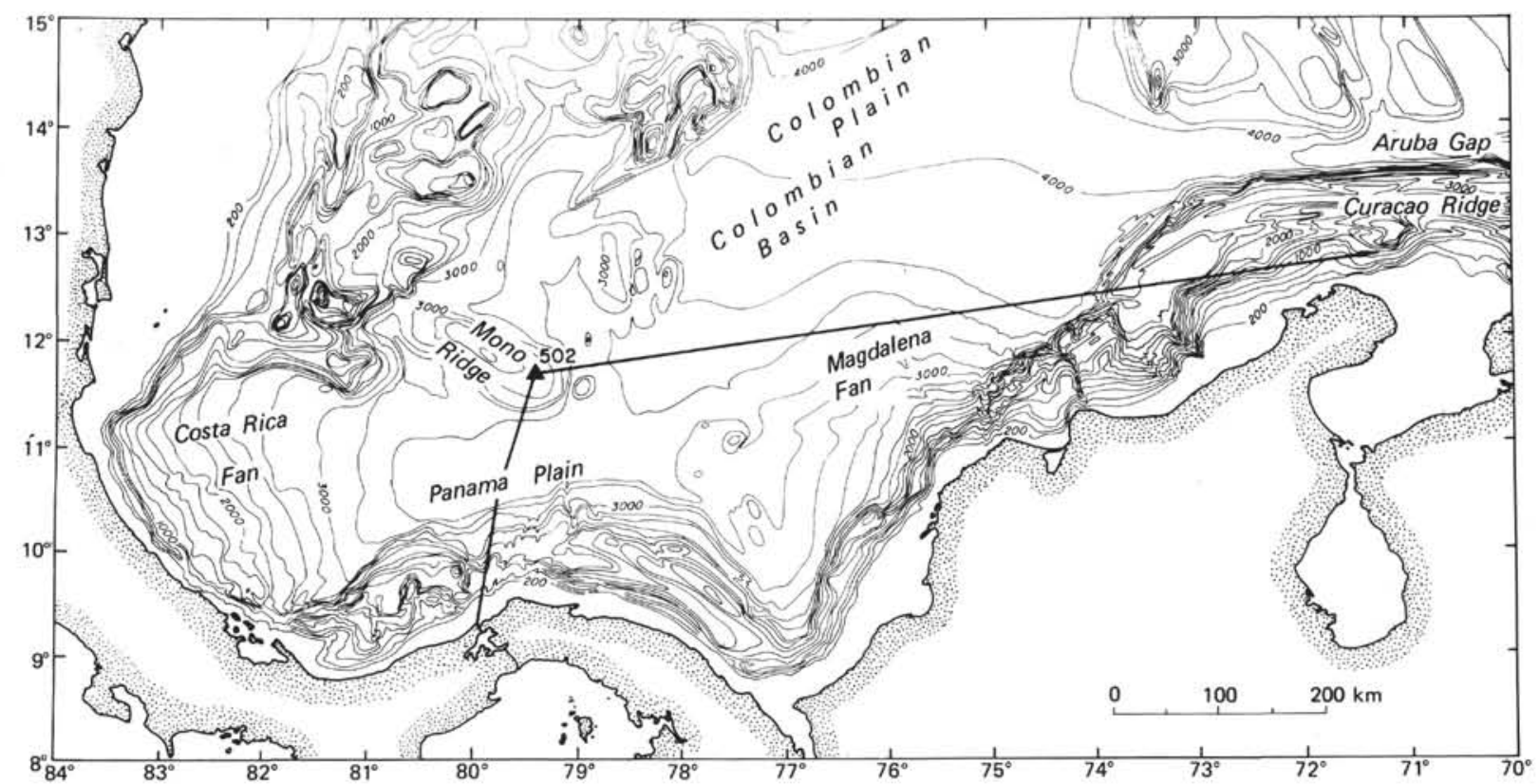

A

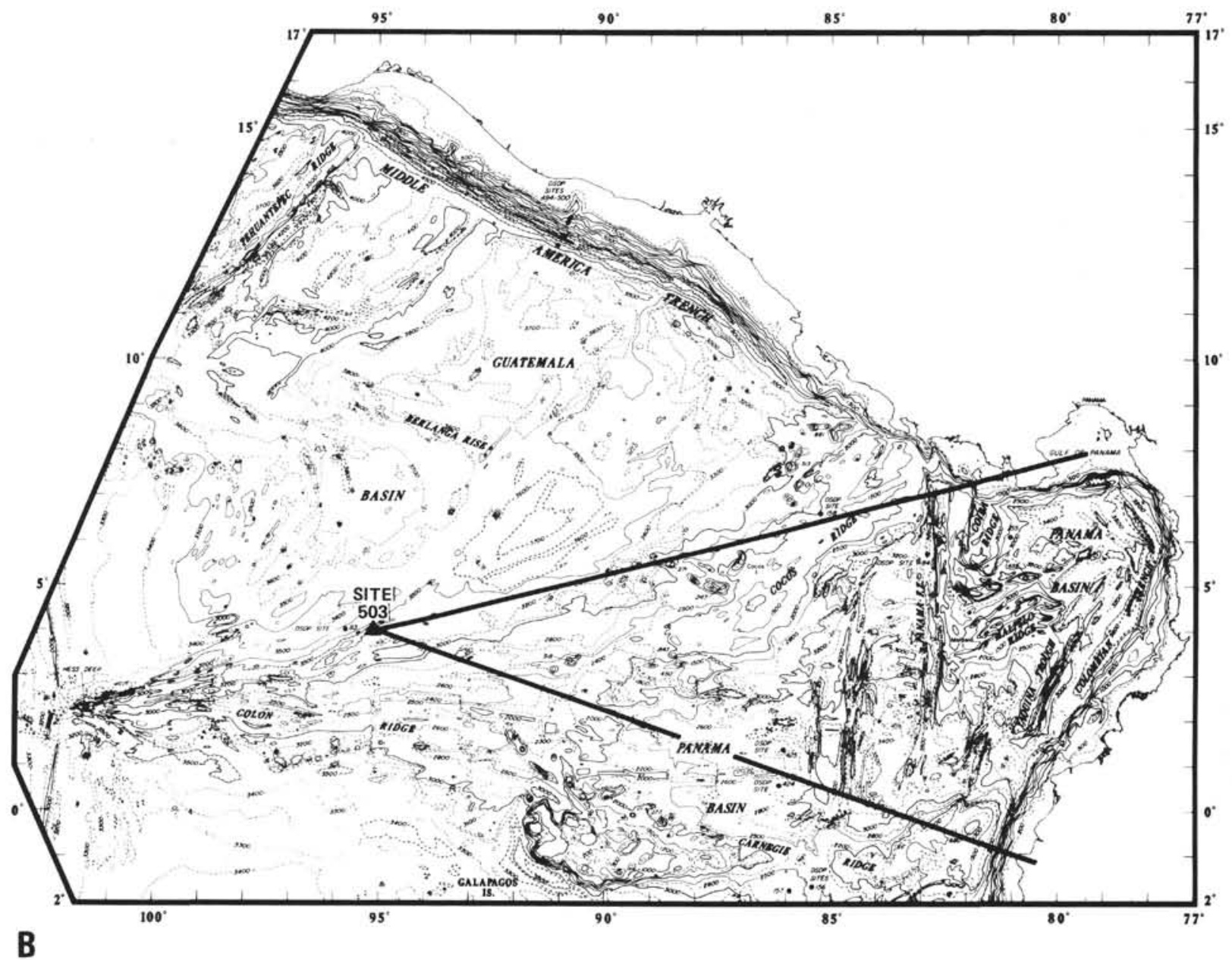

Figure 6. A. Bathymetric chart (depths in meters) of the region of the Caribbean traversed by Glomar Challenger, Leg 68 (after Holcombe, 1975). B. Bathymetric chart (depths in meters) of the region of the Pacific traversed by Leg 68 (after Chase et al., 1970, and Mammerickx et al., 1974). 


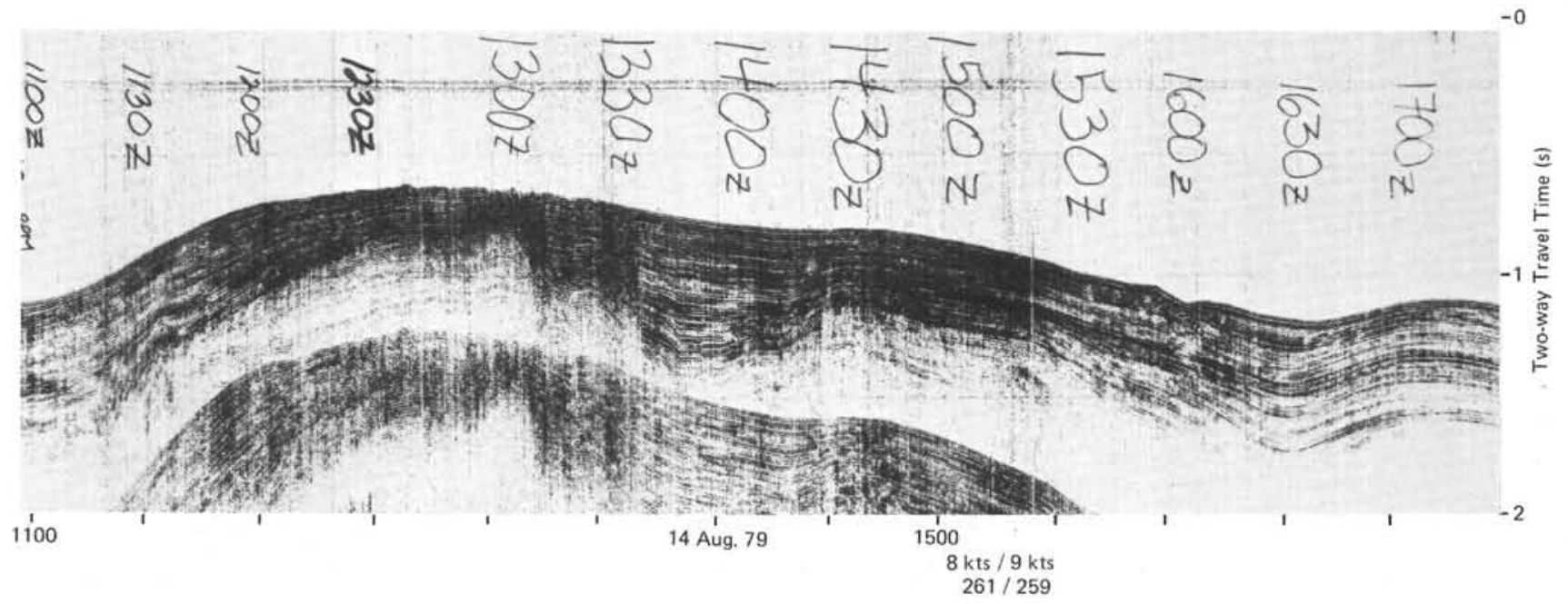

Figure 7. Annotated seismic-reflection profiles collected on Glomar Challenger, Leg 68.

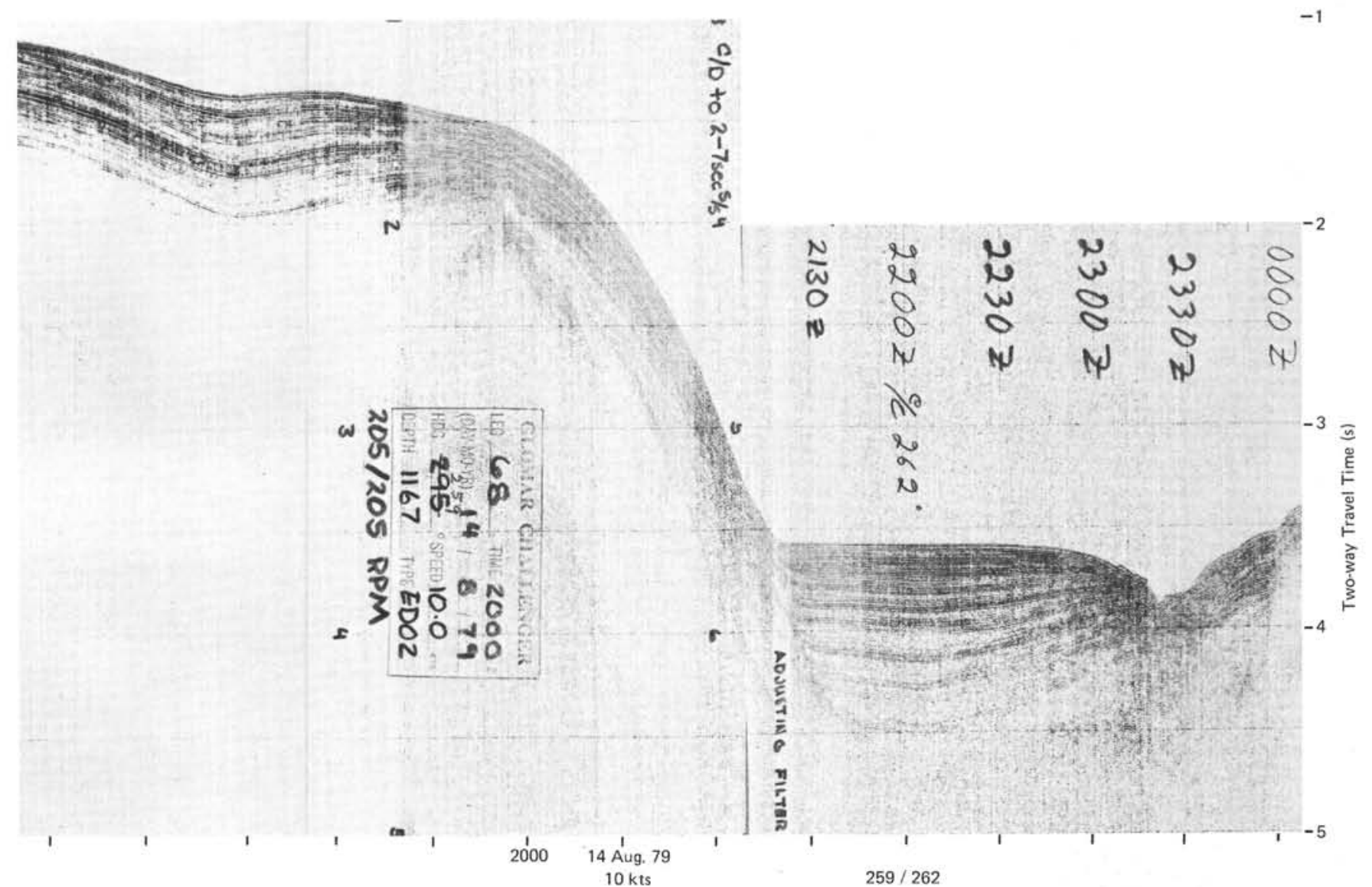

Figure 8. Annotated seismic-reflection profiles collected on Glomar Challenger, Leg 68. 

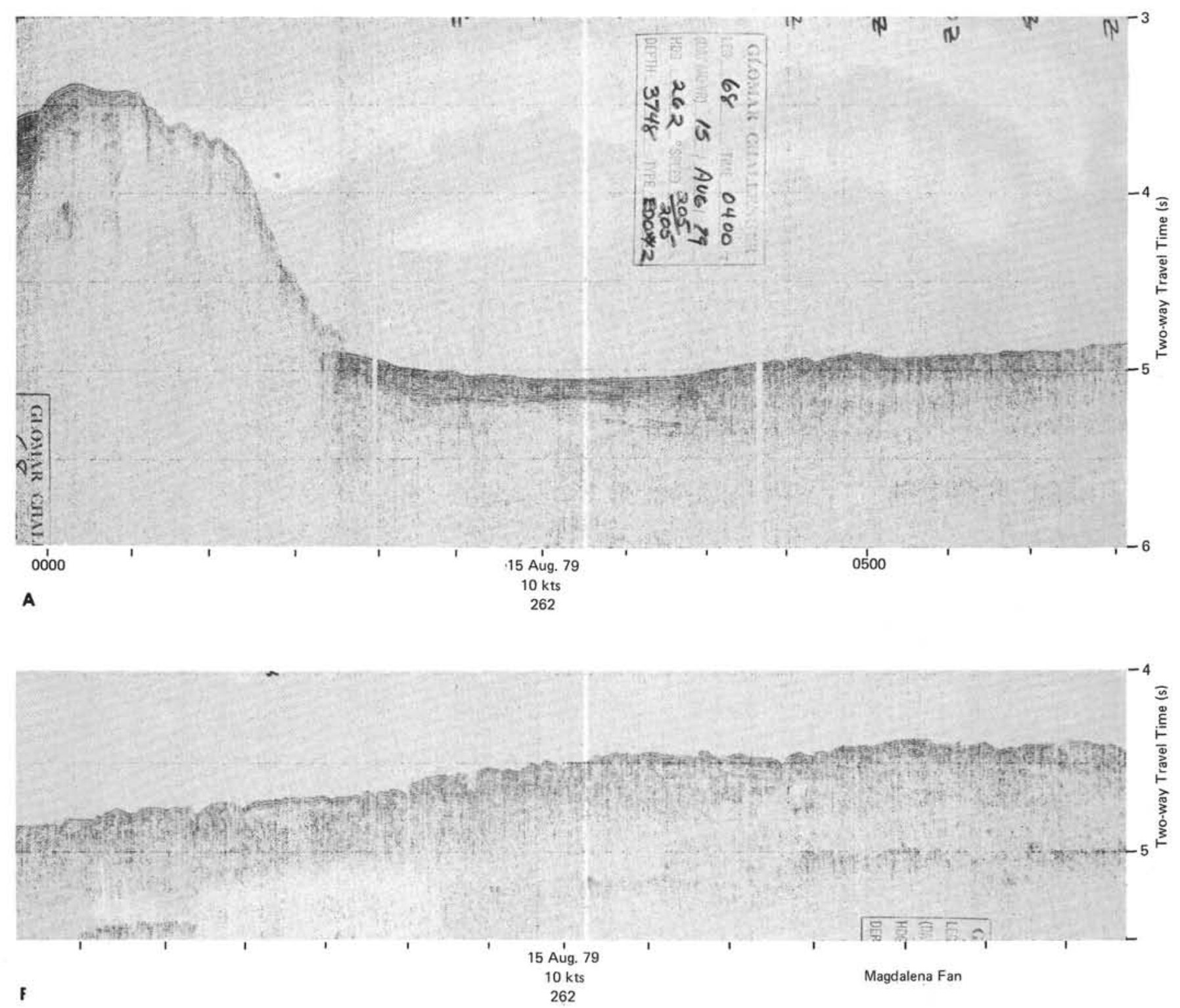

Figure 9. Annotated seismic-reflection profiles collected on Glomar Challenger, Leg 68. 

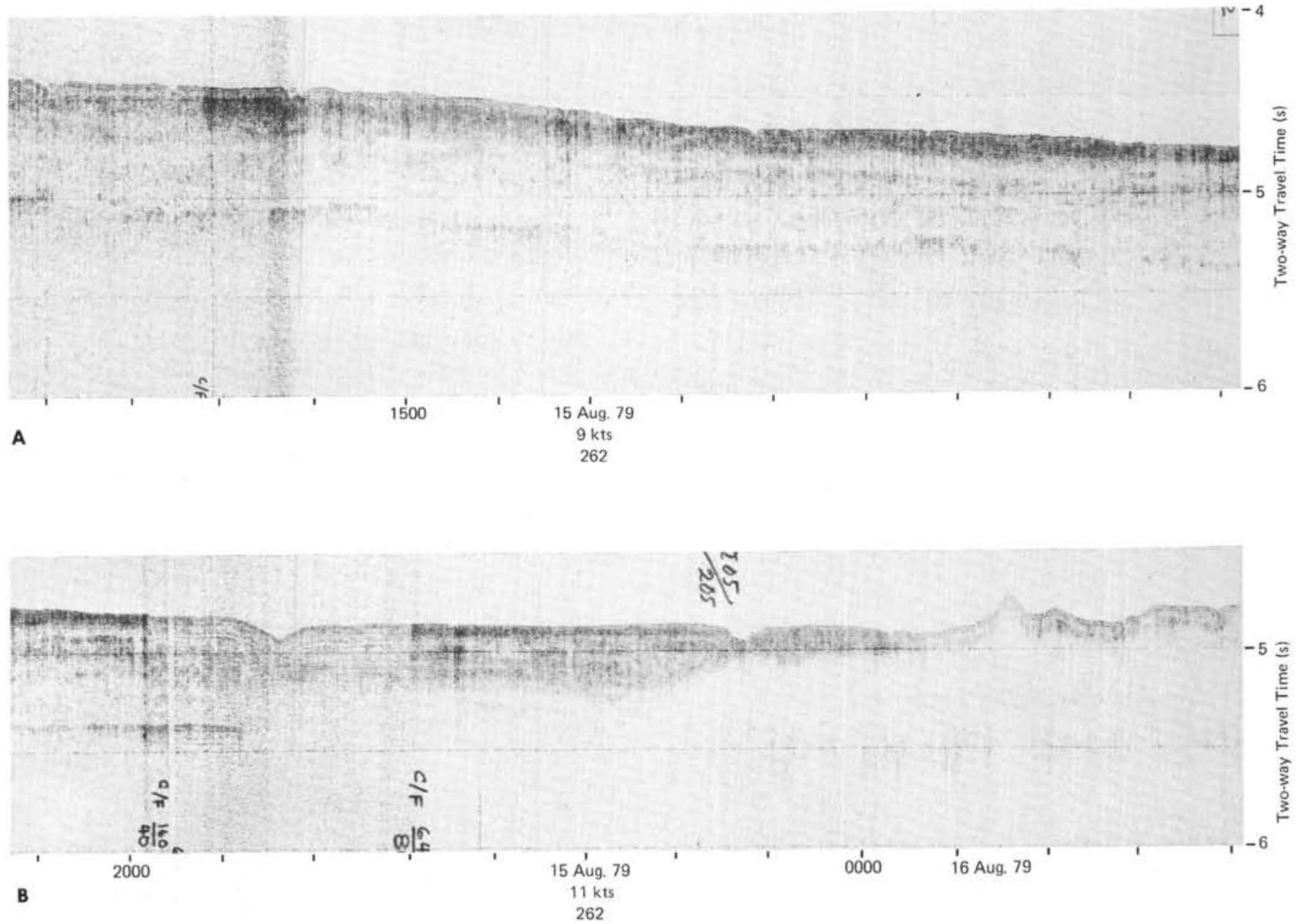

Figure 10. Annotated seismic-reflection profiles collected on Glomar Challenger, Leg 68.

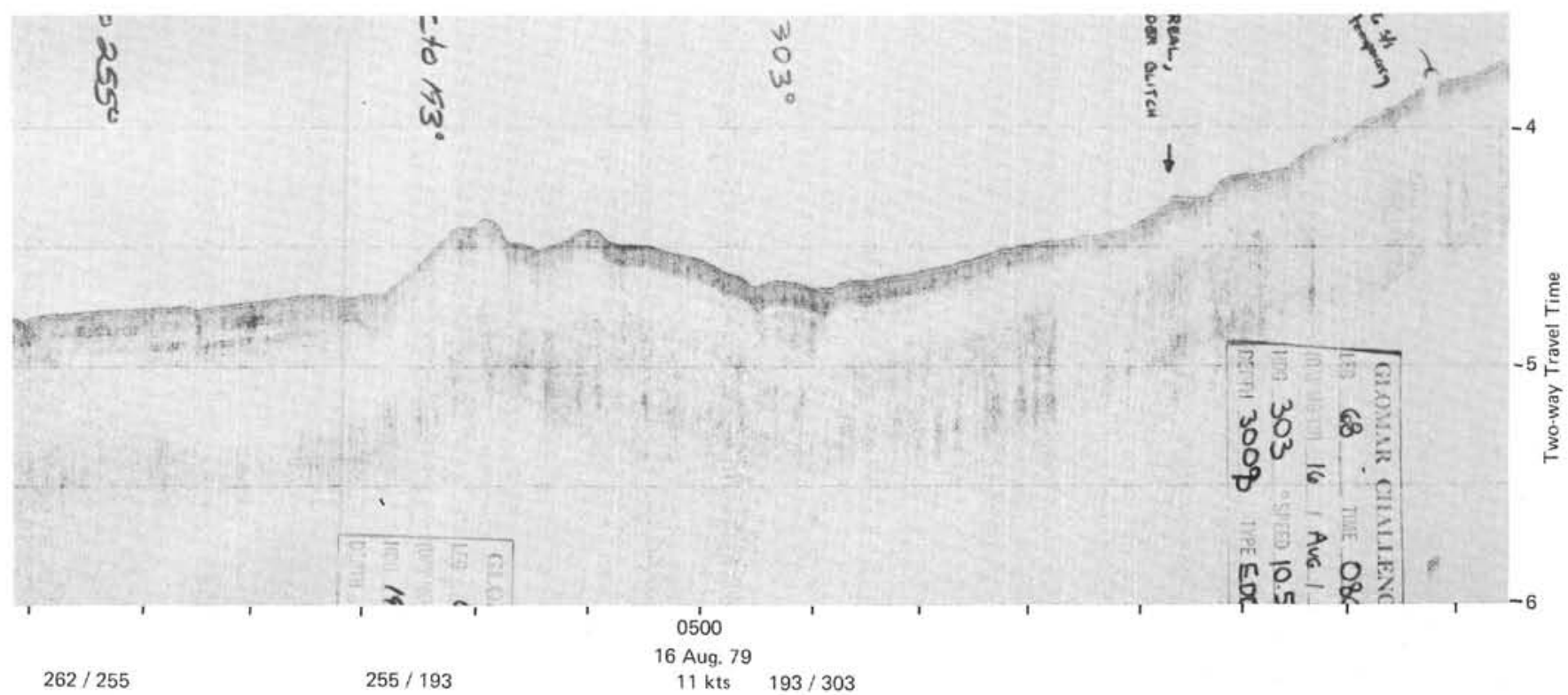

Figure 11. Annotated seismic-reflection profiles collected on Glomar Challenger, Leg 68. 


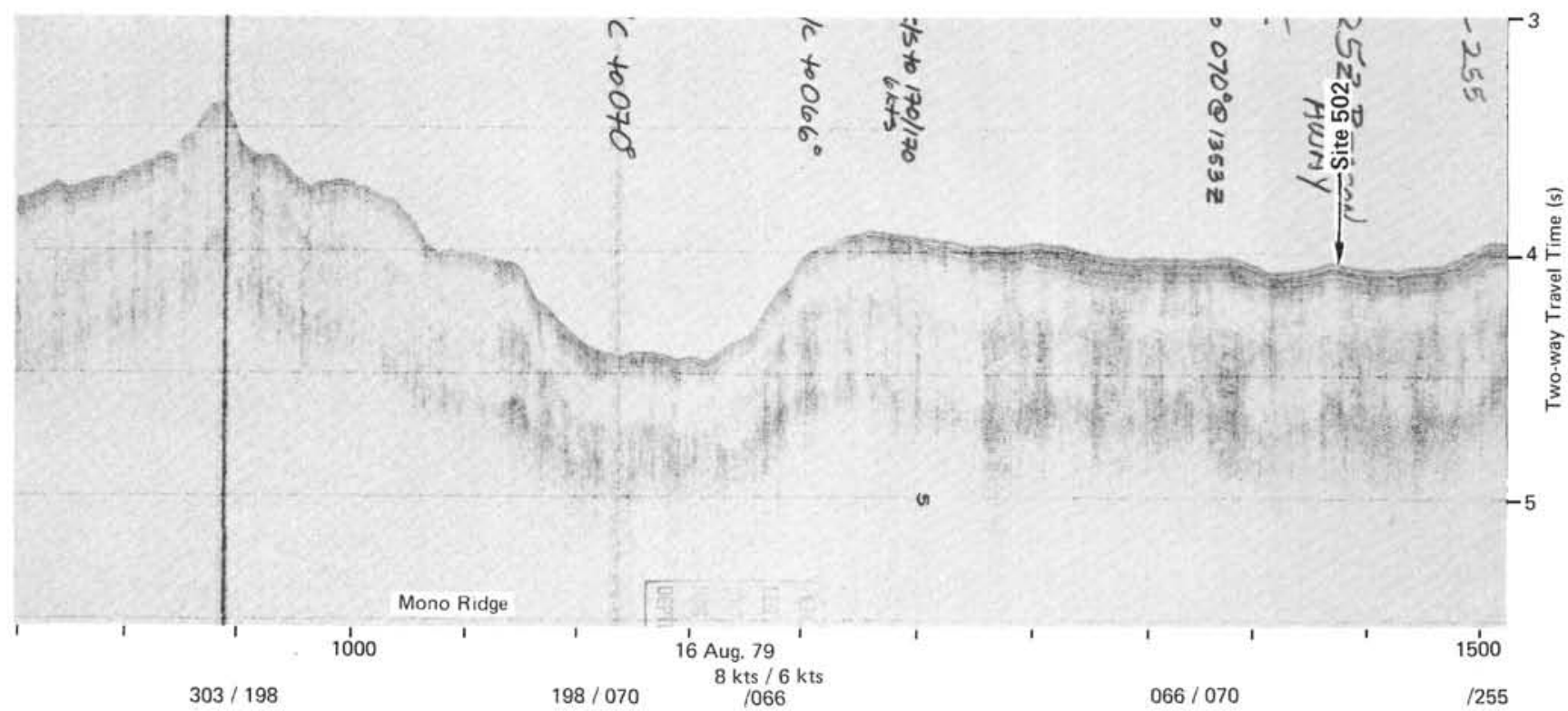

Figure 12. Annotated seismic-reflection profiles collected on Glomar Challenger, Leg 68.

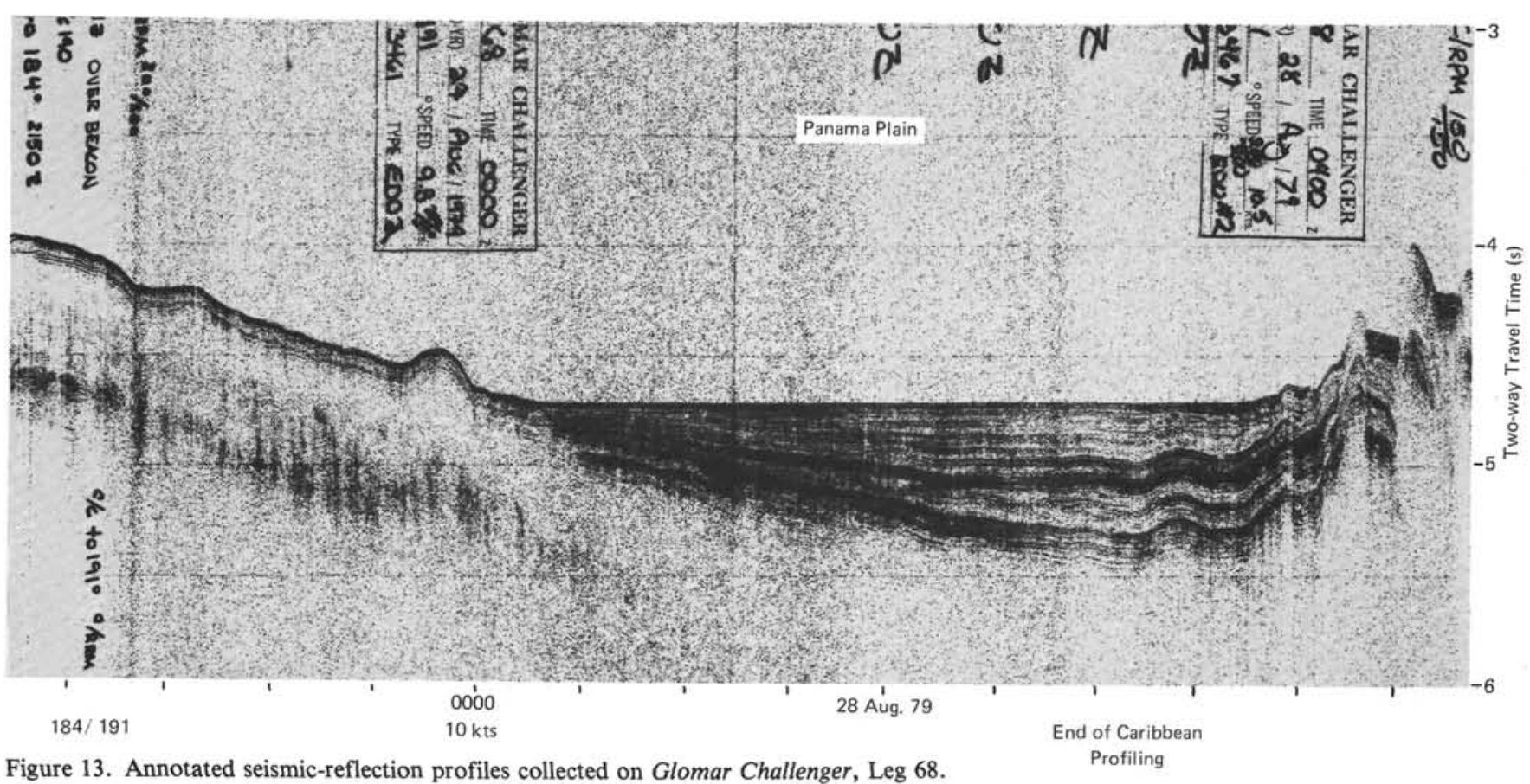




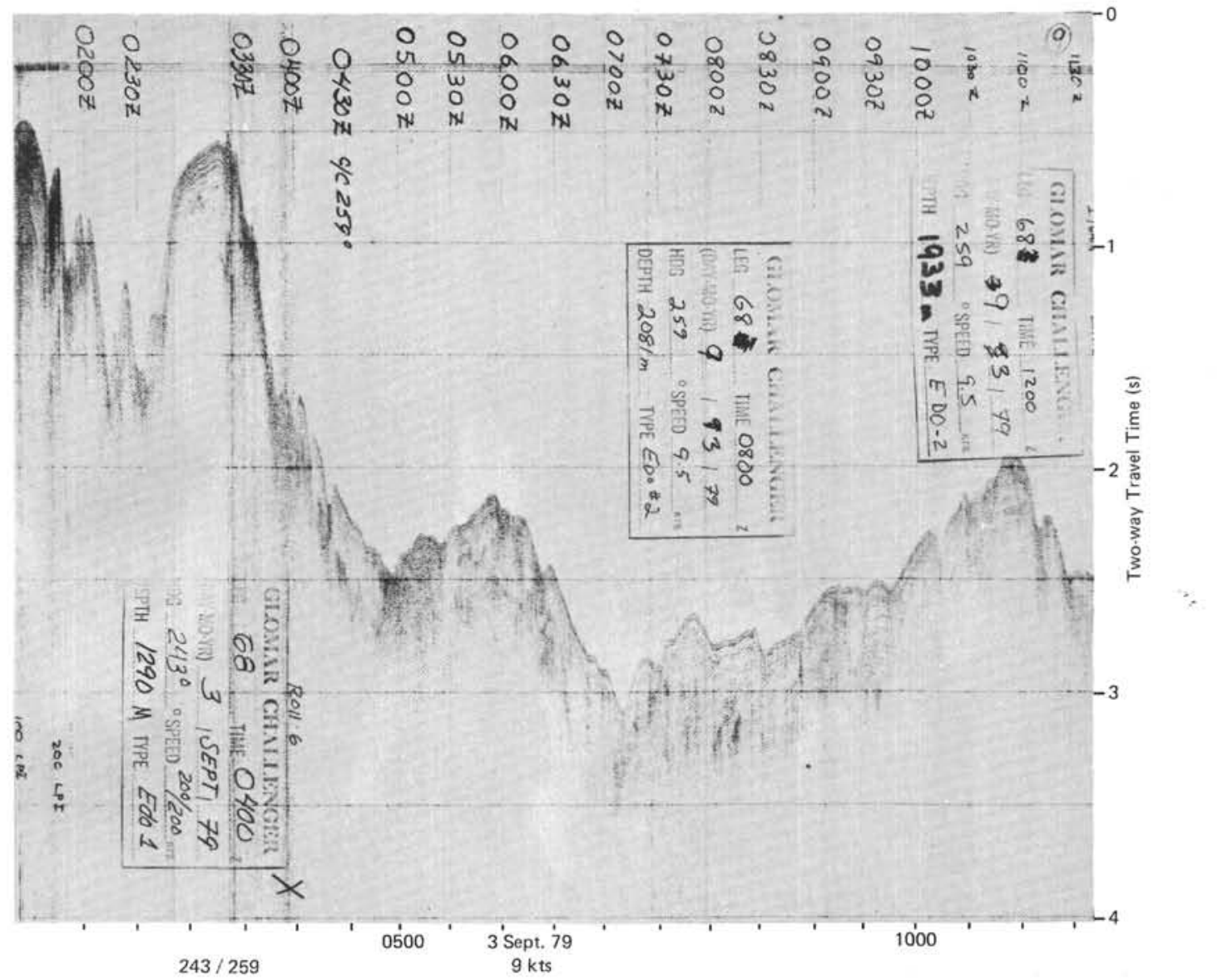

Figure 14. Annotated seismic-reflection profiles collected on Glomar Challenger, Leg 68. 


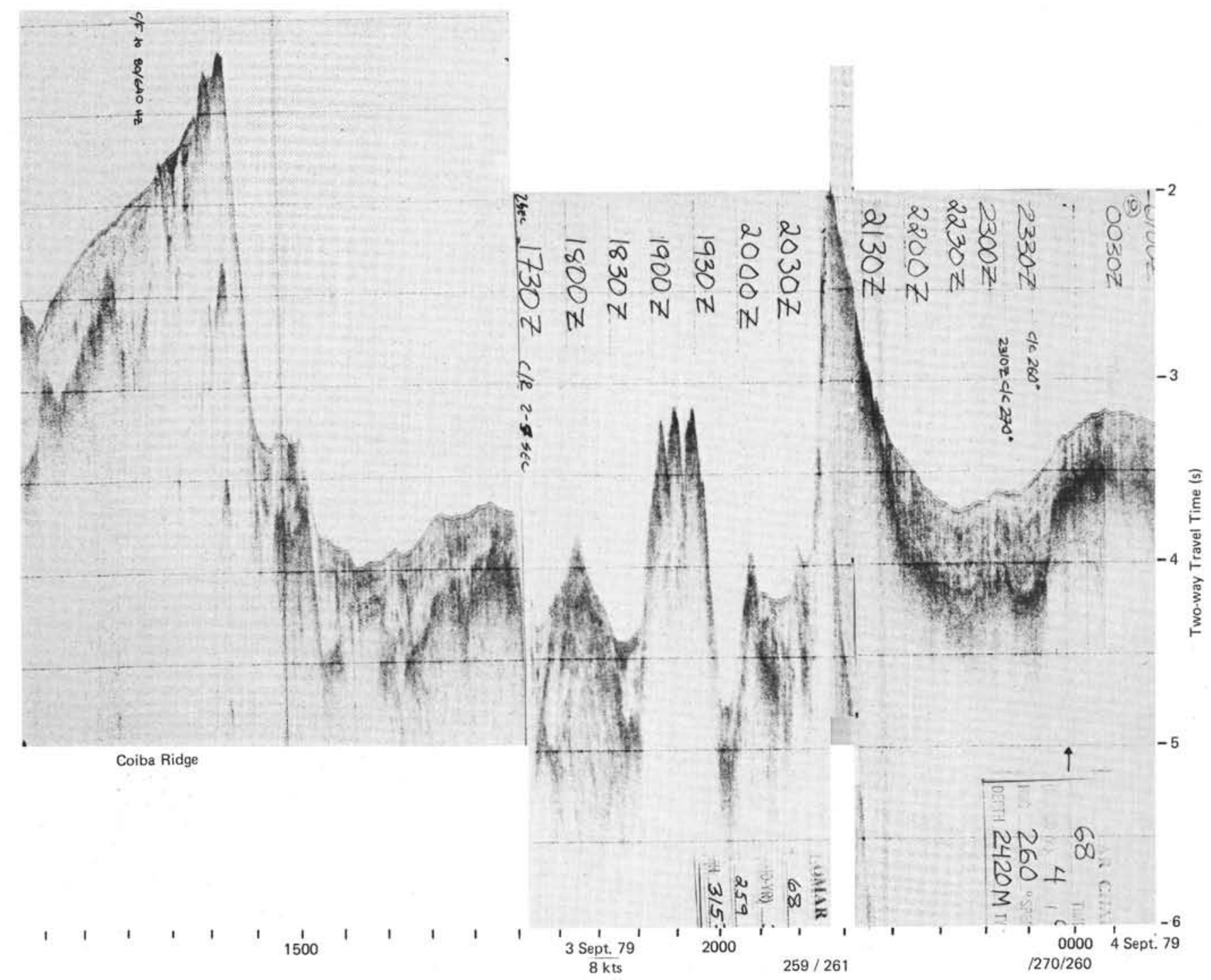

Figure 15. Annotated seismic-reflection profiles collected on Glomar Challenger, Leg 68. 


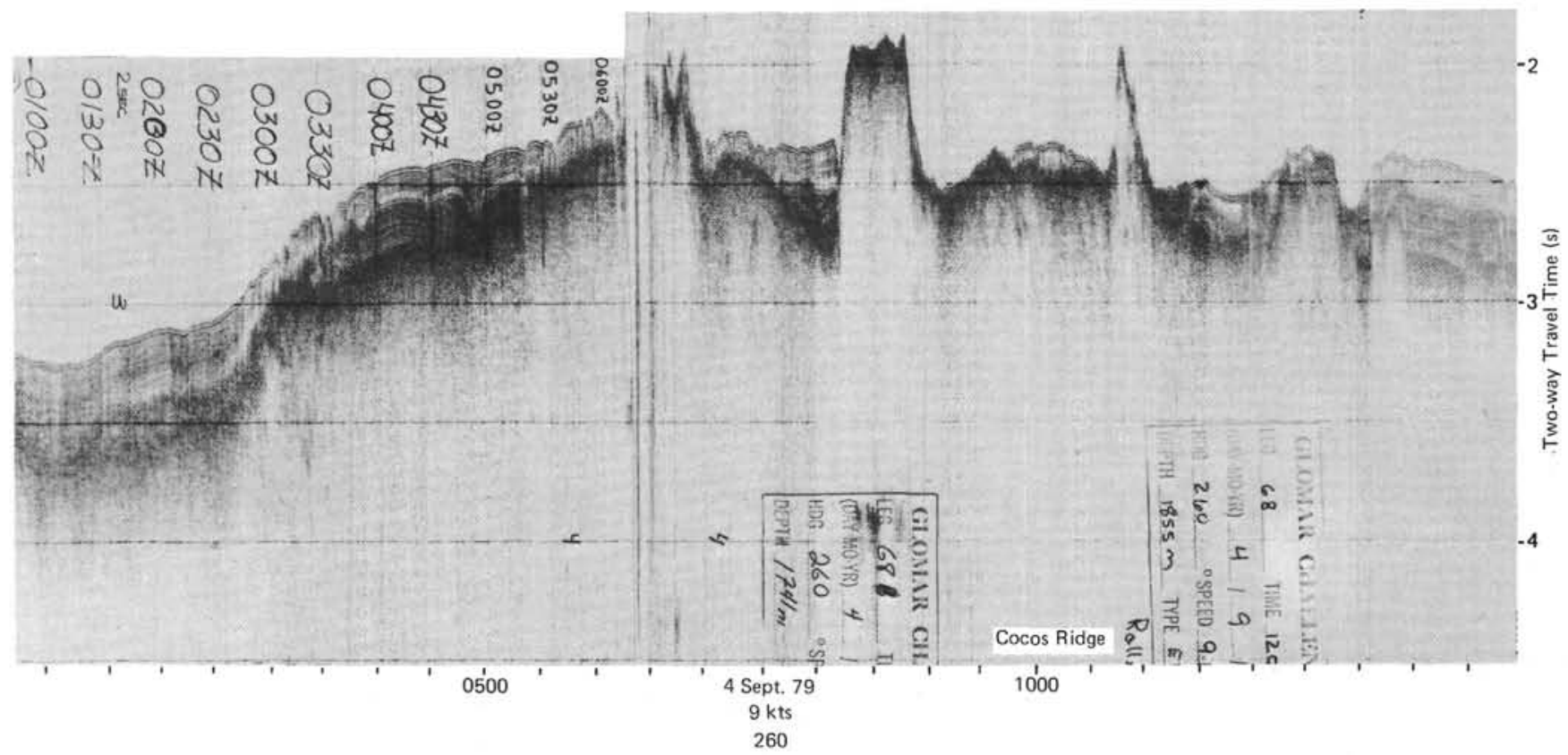

Figure 16. Annotated seismic-reflection profiles collected on Glomar Challenger, Leg 68.

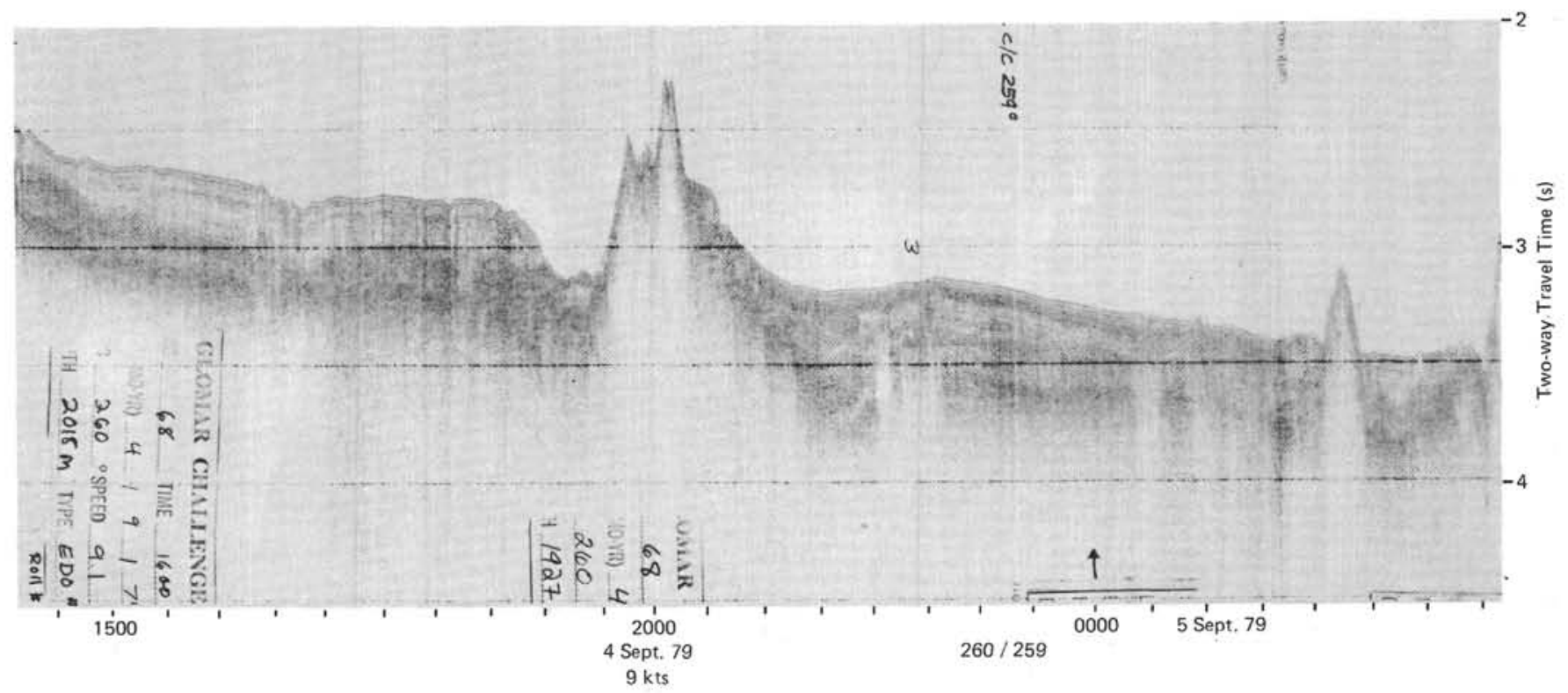

Figure 17. Annotated seismic-reflection profiles collected on Glomar Challenger, Leg 68. 


\section{J. V. GARDNER}

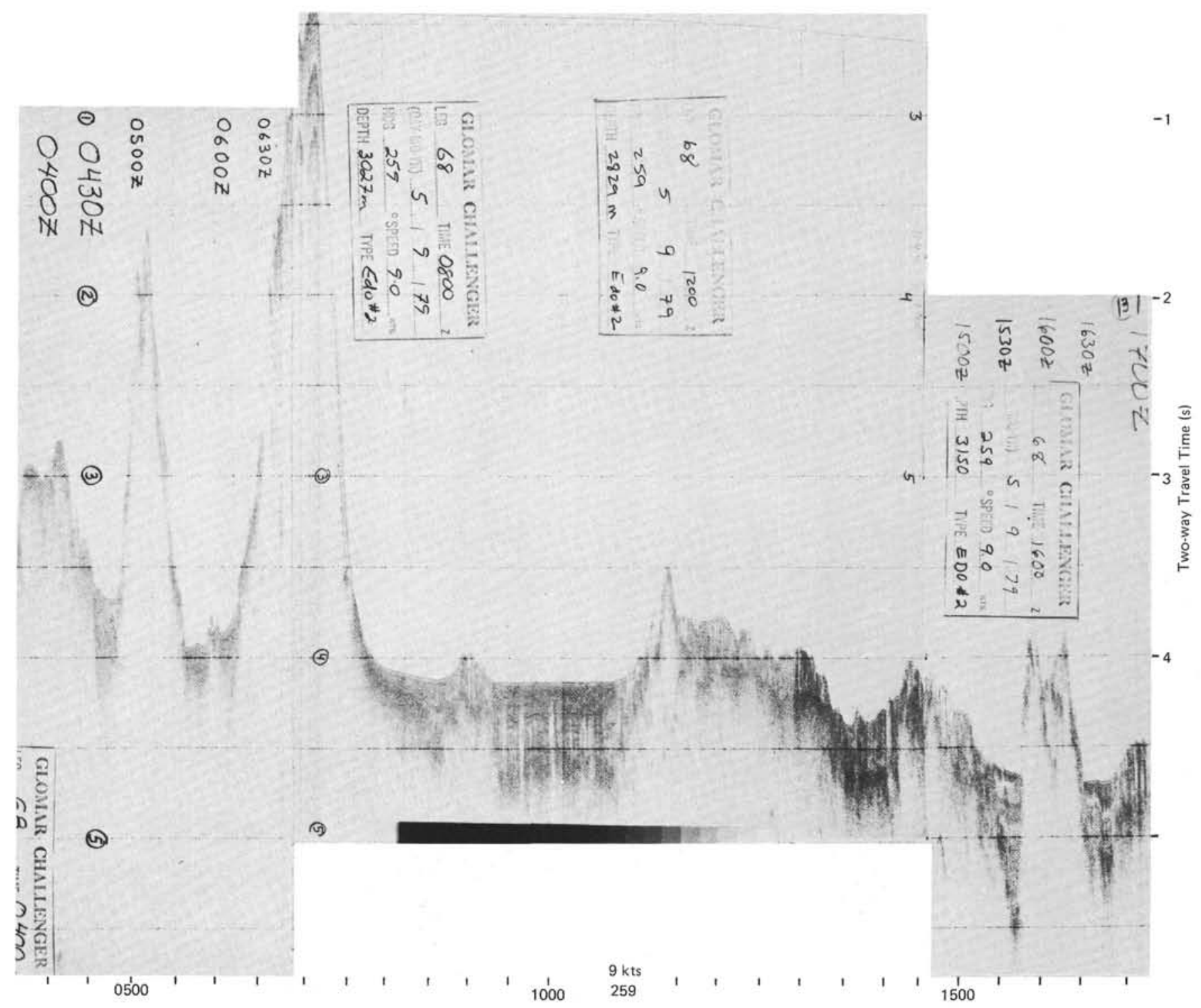

Figure 18. Annotated seismic-reflection profiles collected on Glomar Challenger, Leg 68. 


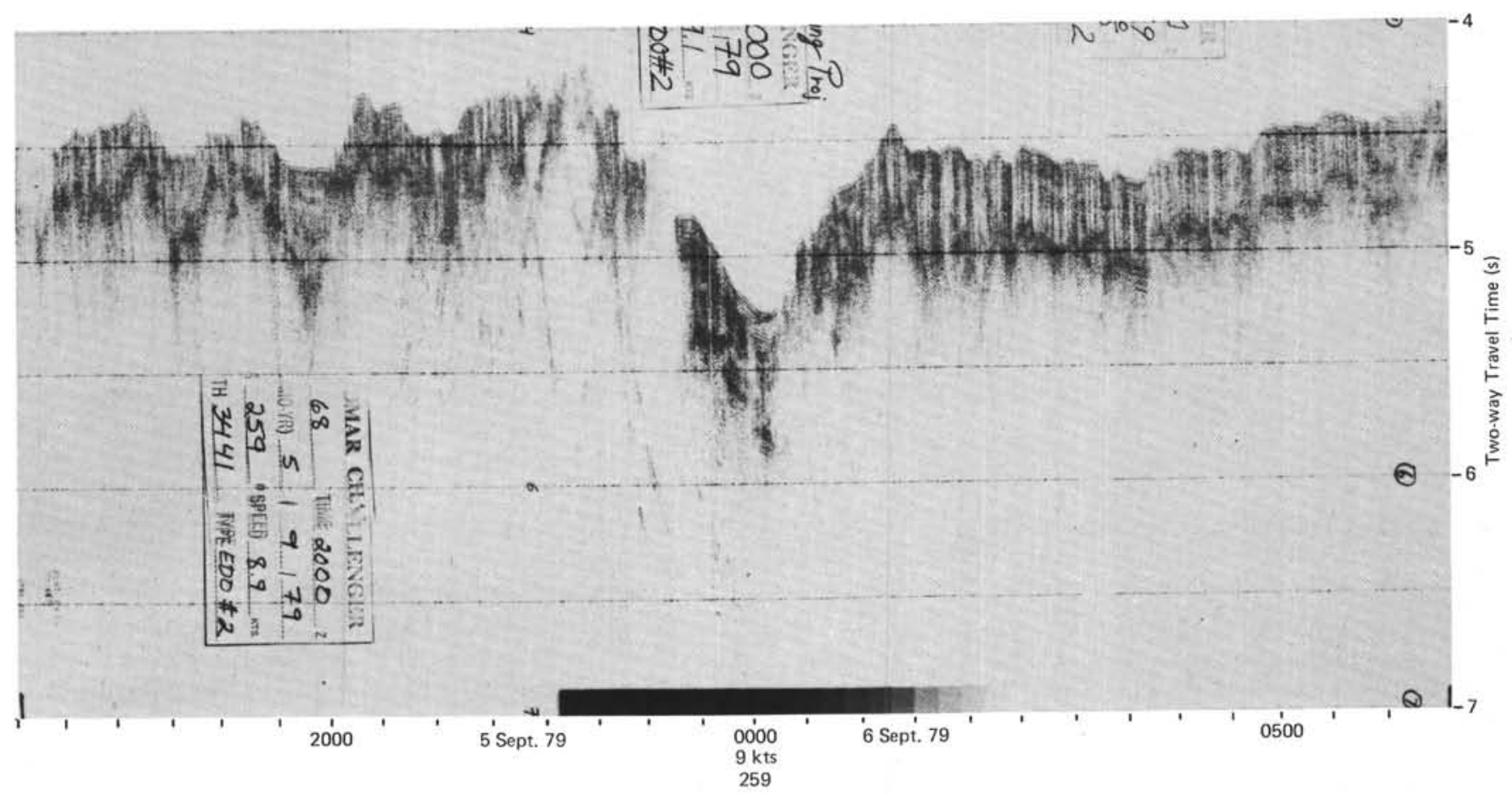

Figure 19. Annotated seismic-reflection profiles collected on Glomar Challenger, Leg 68.

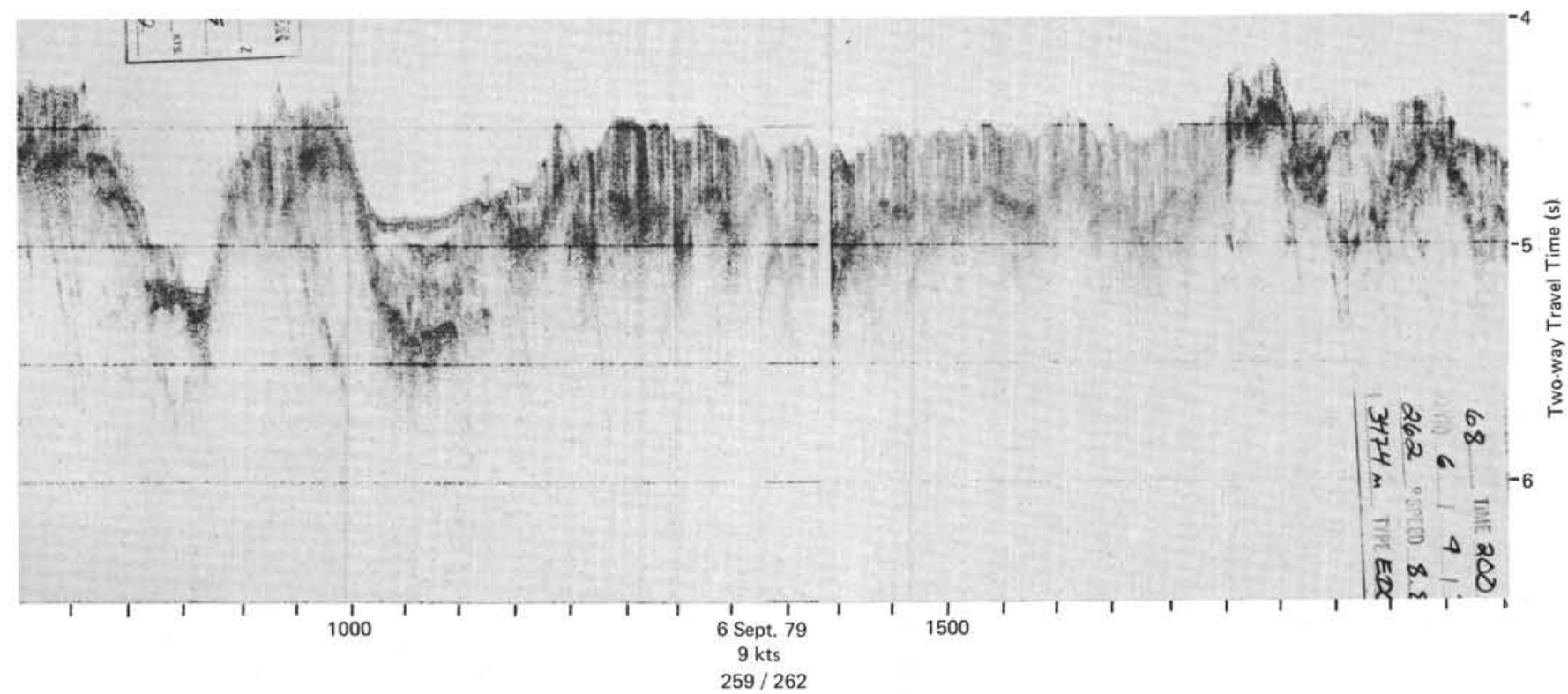

Figure 20. Annotated seismic-reflection profiles collected on Glomar Challenger, Leg 68. 


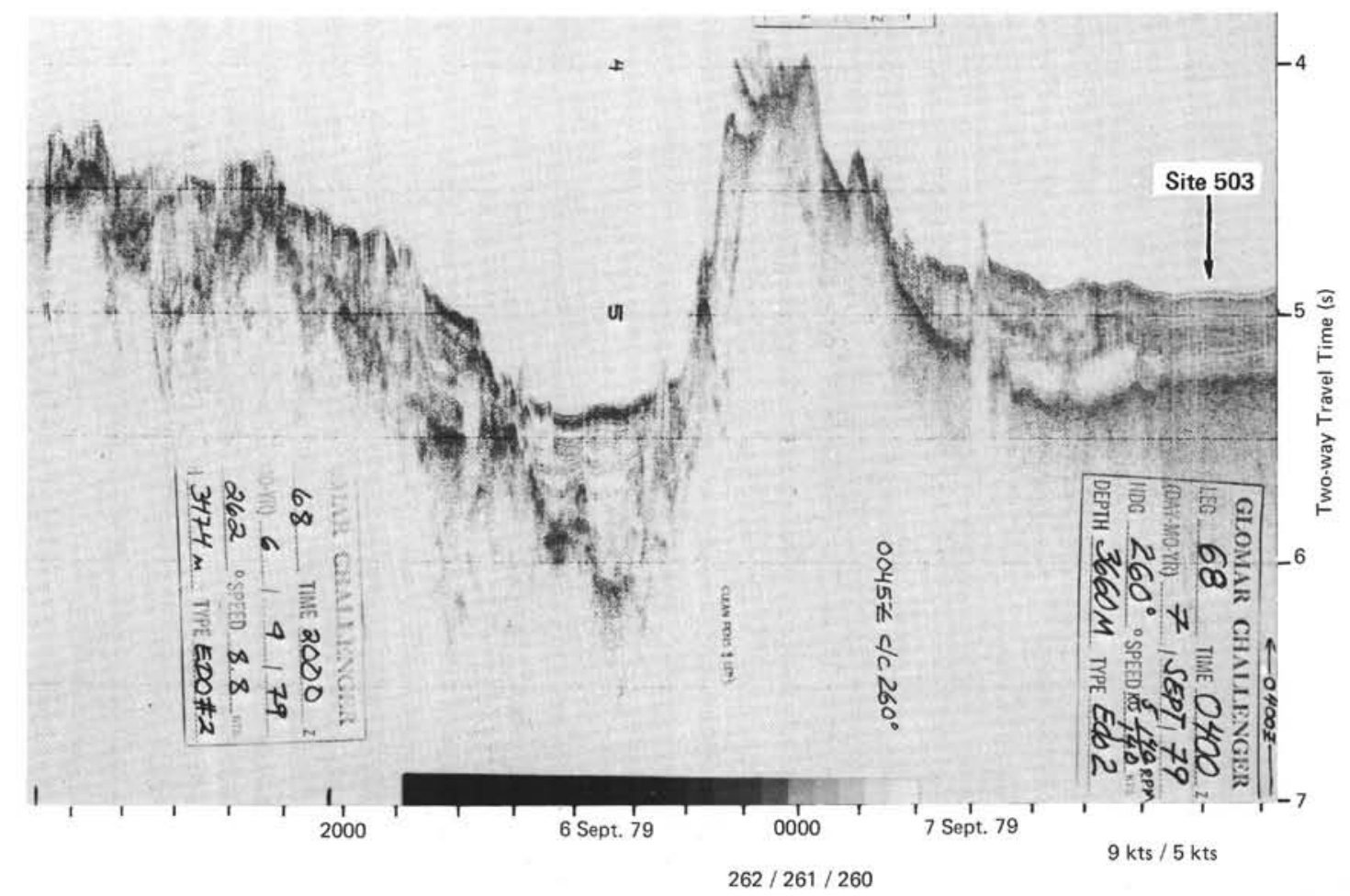

Figure 21. Annotated seismic-reflection profiles collected on Glomar Challenger, Leg 68.

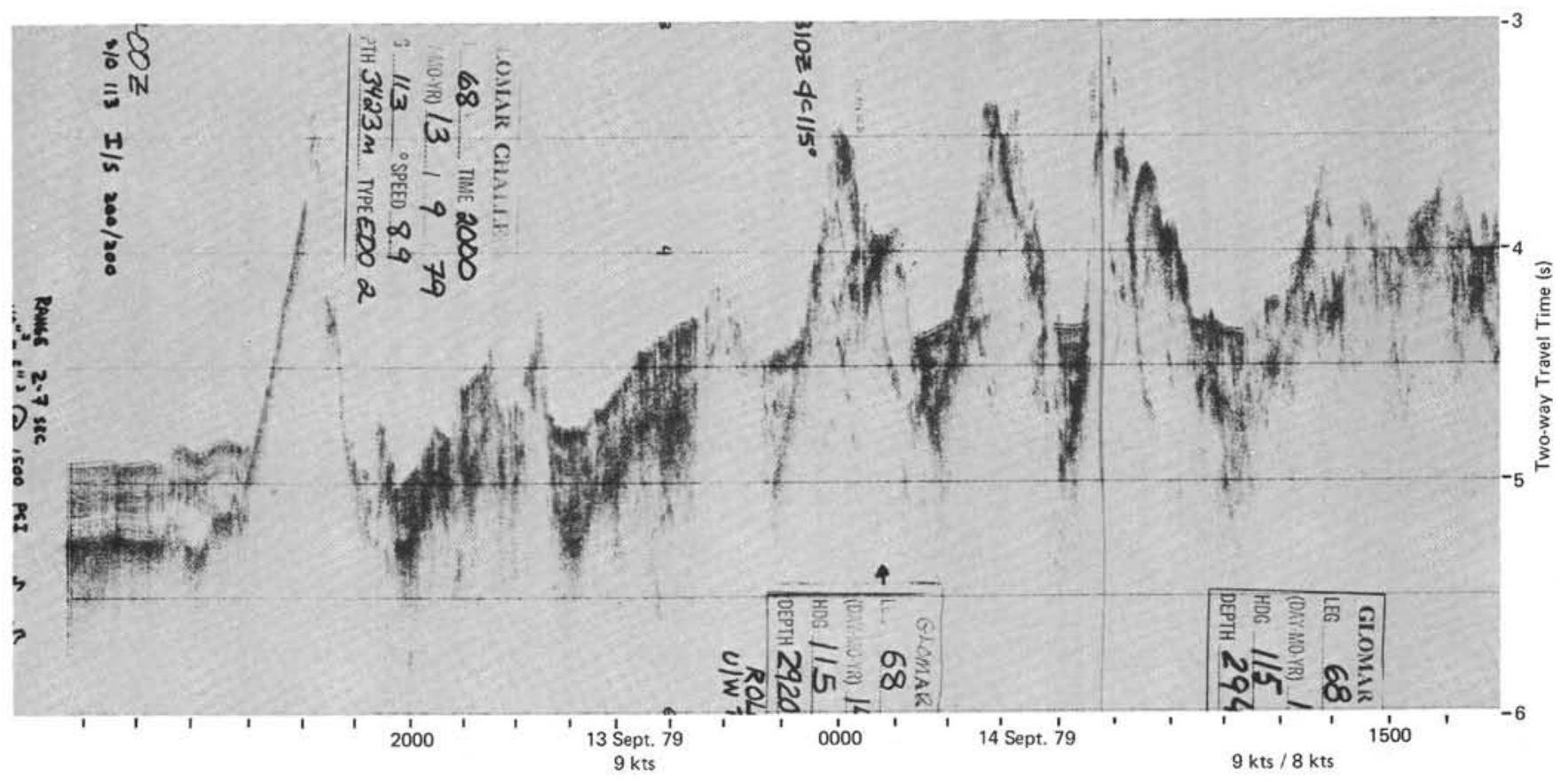

$262 / 113 / 100$

$100 / 115$

Figure 22. Annotated seismic-reflection profiles collected on Glomar Challenger, Leg 68. 


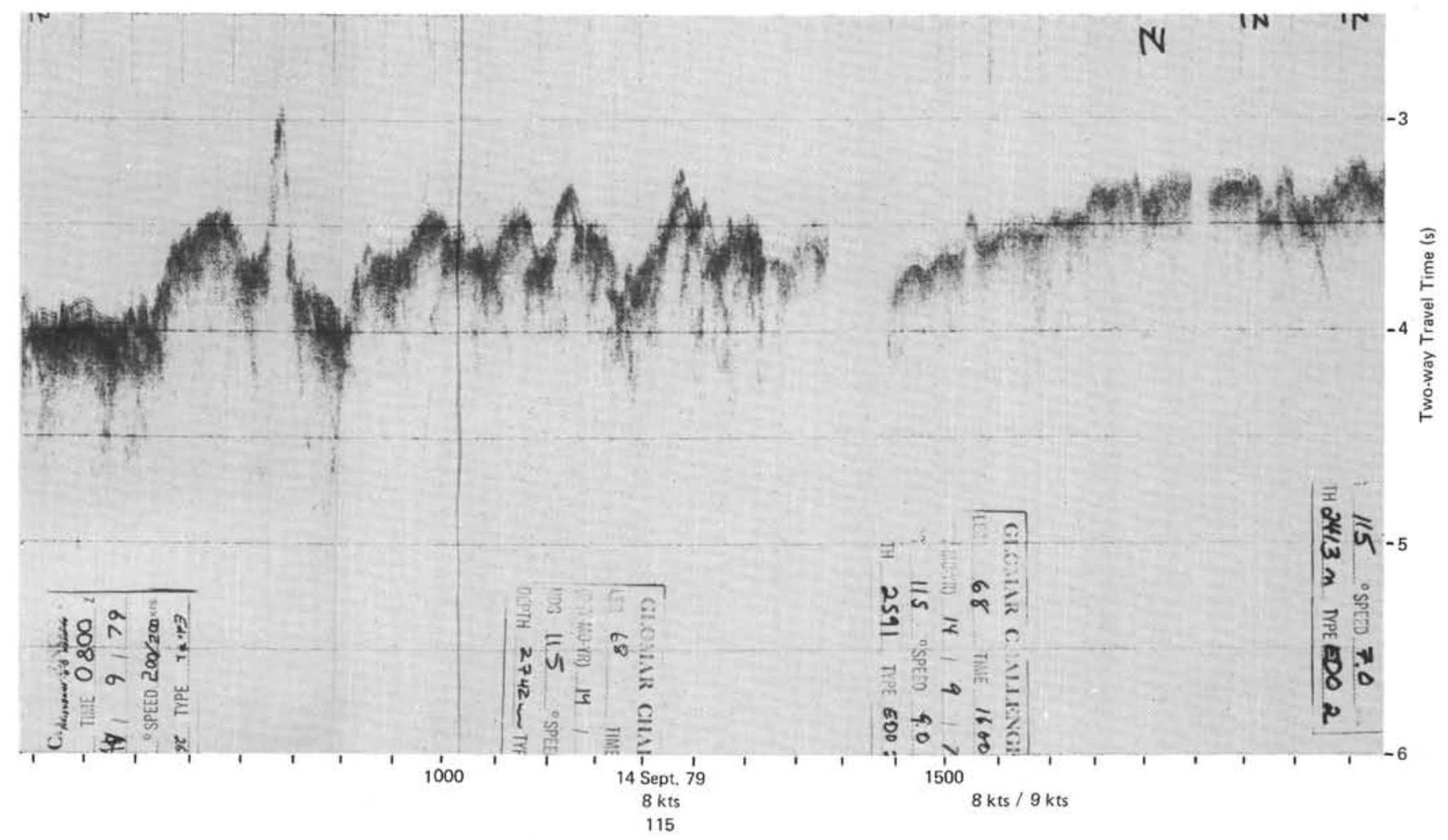

Figure 23. Annotated seismic-reflection profiles collected on Glomar Challenger, Leg 68.

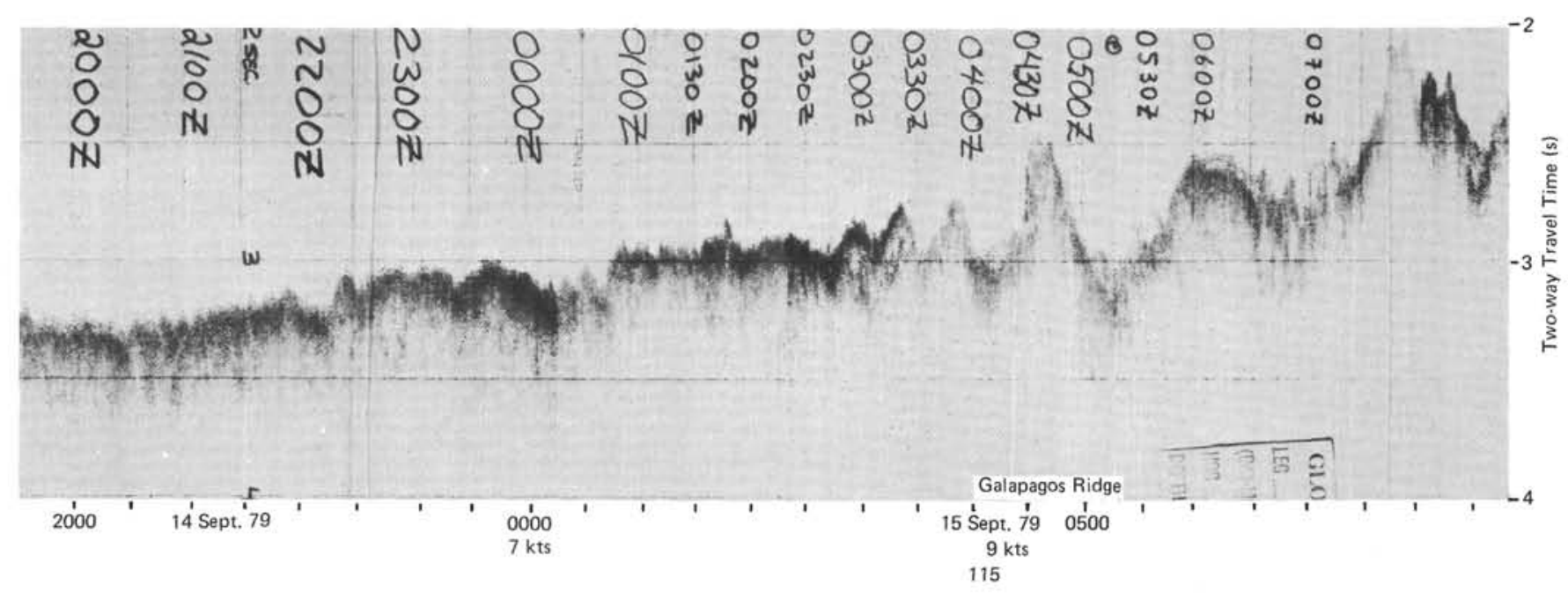

Figure 24. Annotated seismic-reflection profiles collected on Glomar Challenger, Leg 68. 


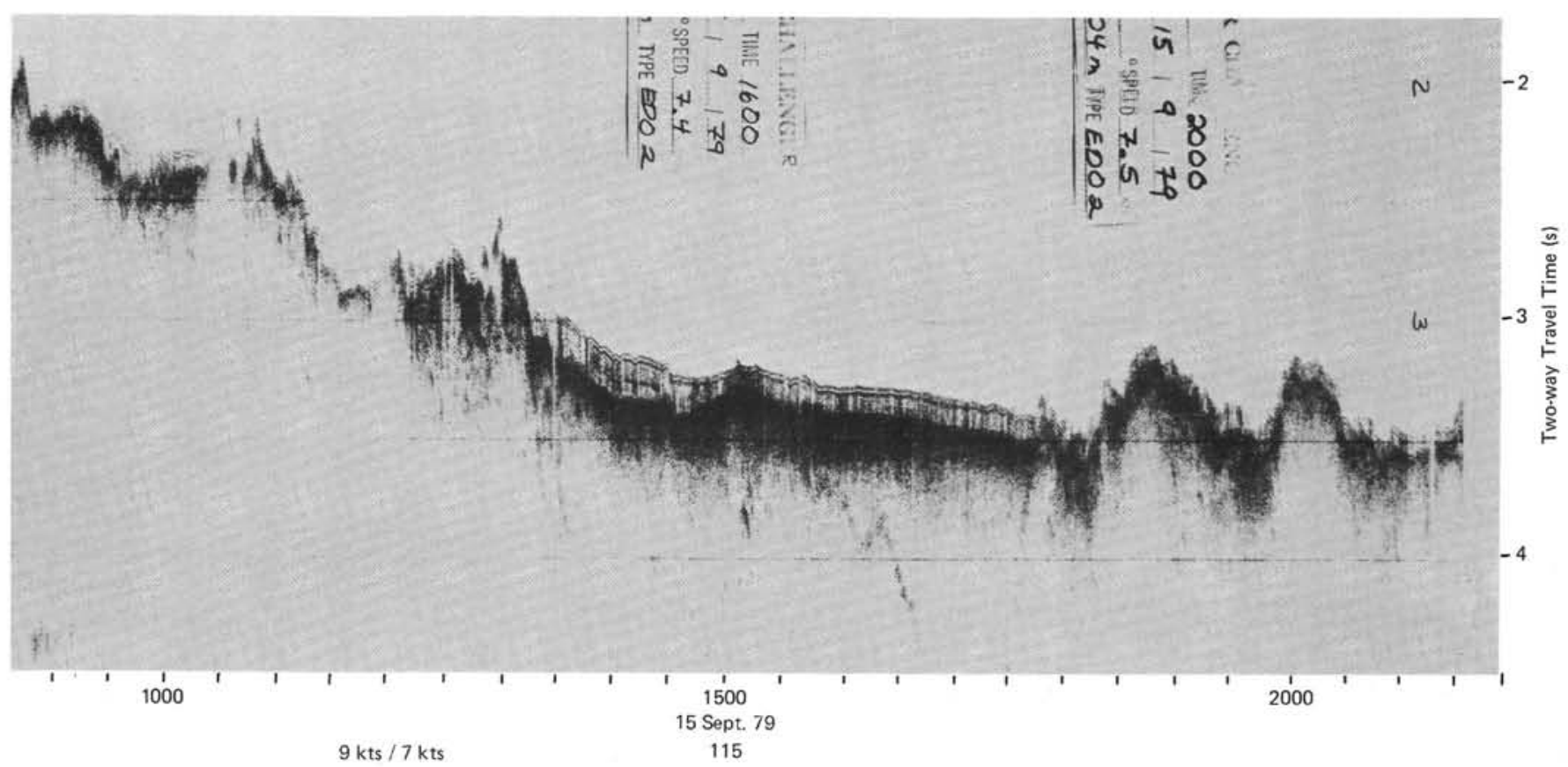

Figure 25. Annotated seismic-reflection profiles collected on Glomar Challenger, Leg 68.
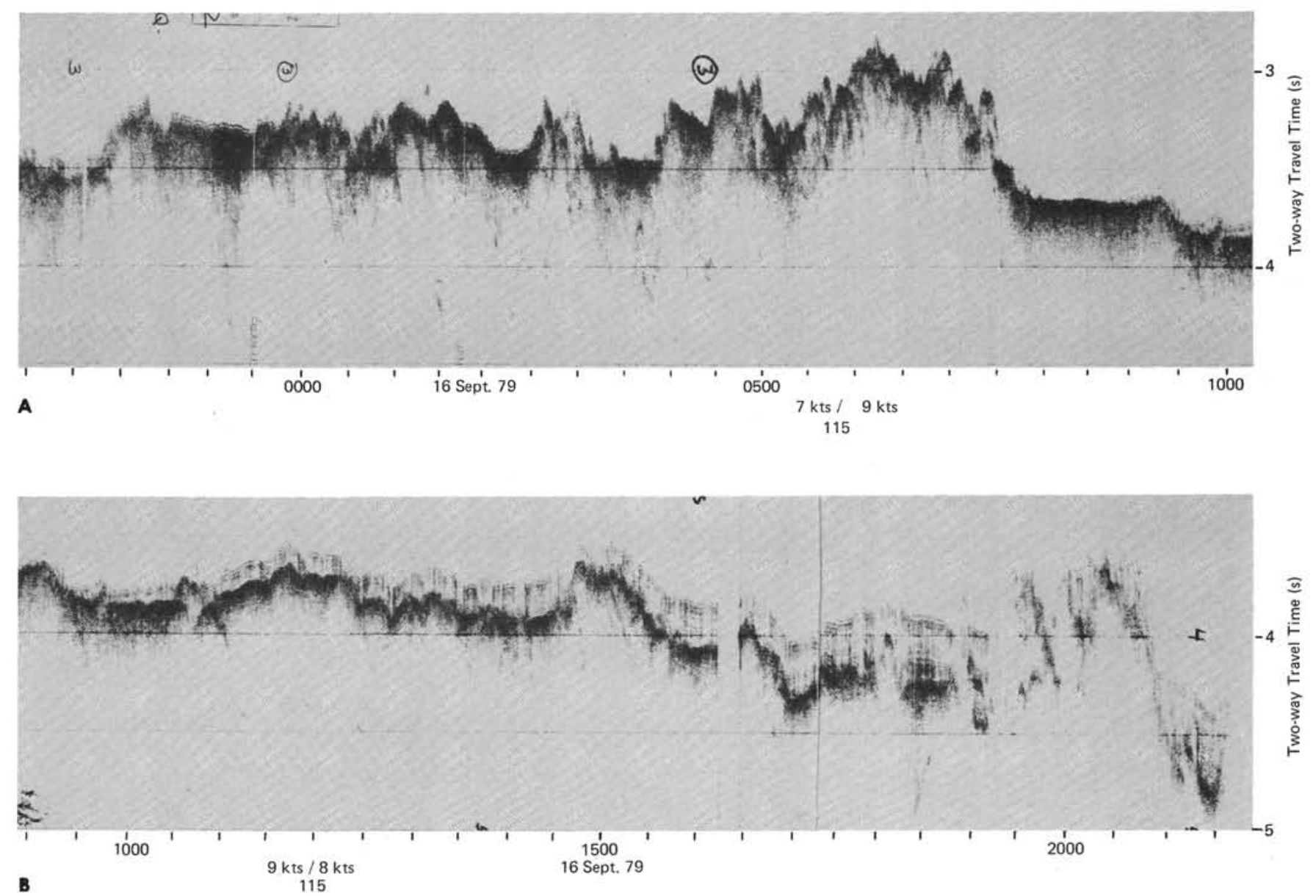

Figure 26. Annotated seismic-reflection profiles collected on Glomar Challenger, Leg 68. 


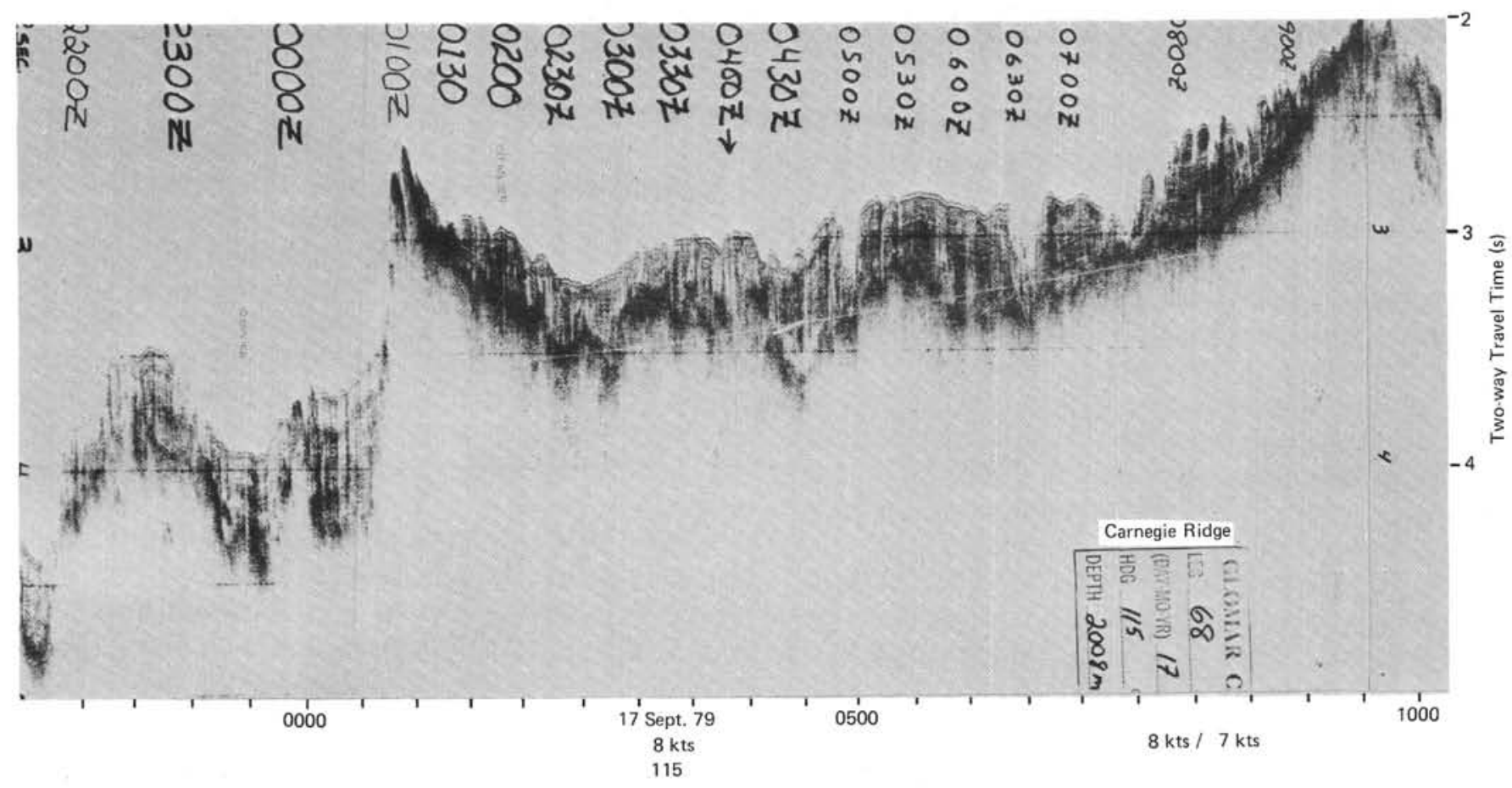

Figure 27. Annotated seismic-reflection profiles collected on Glomar Challenger, Leg 68.

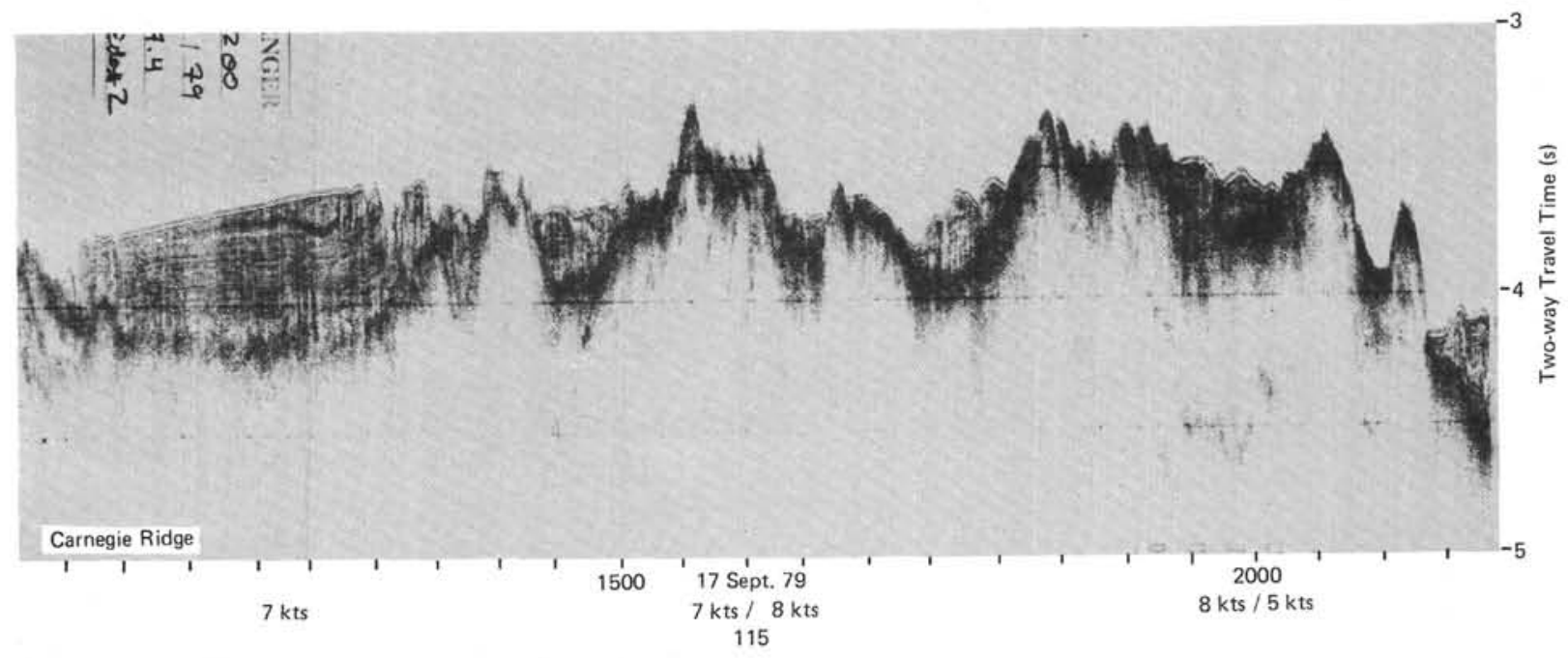

Figure 28. Annotated seismic-reflection profiles collected on Glomar Challenger, Leg 68. 
J. V. GARDNER

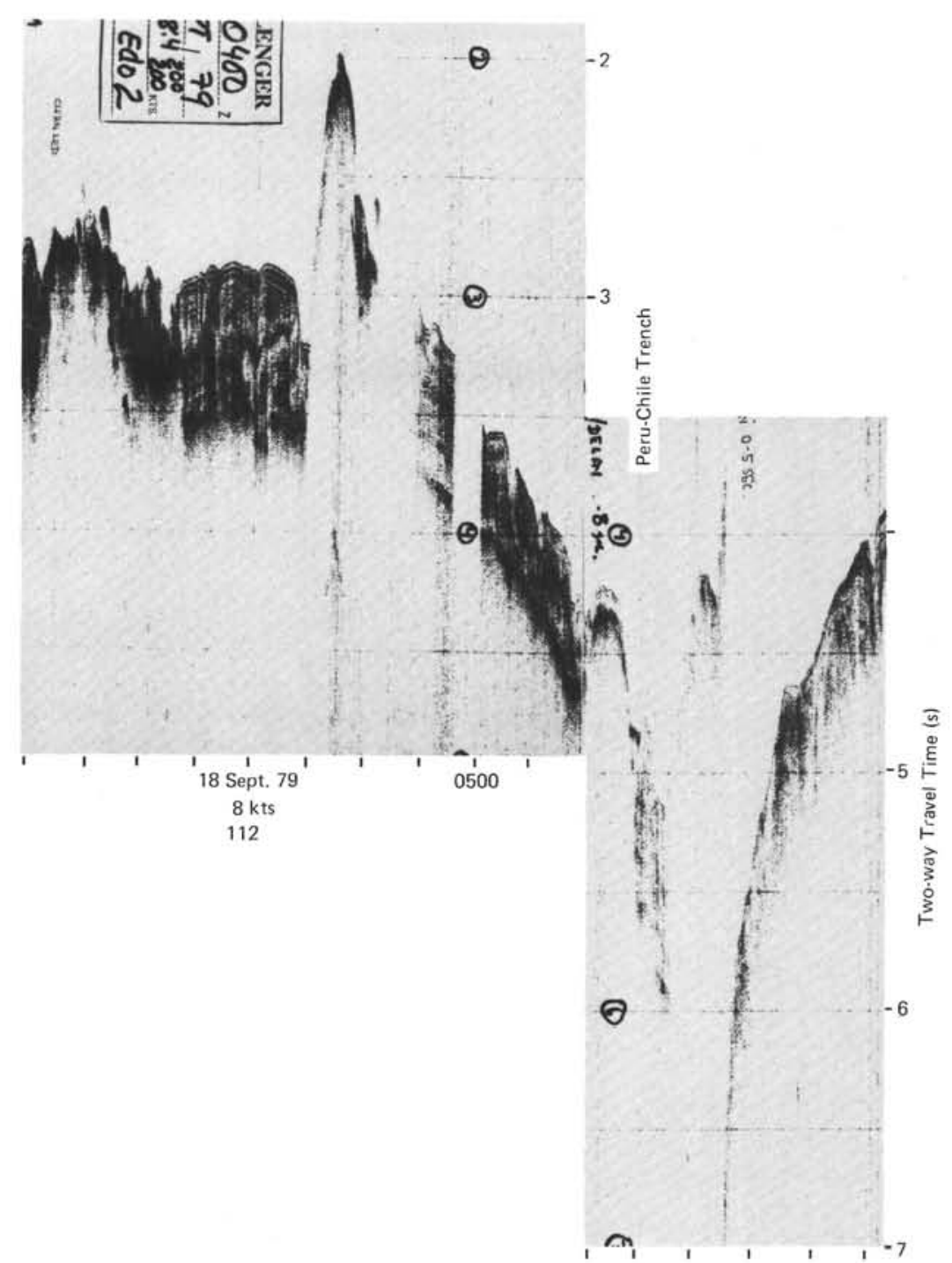

Figure 29. Annotated seismic-reflection profiles collected on Glomar Challenger, Leg 68. 